SHORT PULSE HIGH POWER MICROWAVE SURFACE FLASHOVER

by

LUKE MILTON MCQUAGE, B.S.E.E

A THESIS

IN

\title{
ELECTRICAL ENGINEERING
}

Submitted to the Graduate Faculty of Texas Tech University in

Partial Fulfillment of

the Requirements for

the Degree of

MASTER OF SCIENCE

IN

\section{ELECTRICAL ENGINEERING}

Approved

Dr. Andreas Neuber

Chairperson of the Committee

Hermann Krompholz

Fred Hartmeister

Dean of the Graduate School

December, 2008 
(C) 2008

Luke McQuage

All Rights Reserved 


\section{ACKNOWLEDGMENTS}

I would like to thank Drs. Neuber and Krompholz for serving on my committee and their invaluable help and guidance. A special thanks goes to Dr. Neuber for his patience and encouragement during periods of sluggish productivity and frustration with the project. I would also like to thank the staff at Texas Tech, especially Danny Garcia, Elmer Thorton, Dino Castro, Shannon Gray, Joe Copeland and Marie Byrd. Additionally, I would like to thank all of my colleagues and the faculty at the Center for Pulsed Power and Power Electronics for listening to my manifold complaints and problems and their many suggestions and advice, specifically John Walter, Dr. Mankowski, Dr. Edmiston, Dr. Krile, Dr. Hatfield, and Jack Chen. A special thanks also goes to the Air Force Office of Scientific Research for funding this project. Lastly, I would like to thank my parents and brothers for their love and support, without which none of this would have been possible. 


\section{TABLE OF CONTENTS}

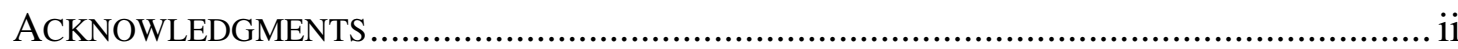

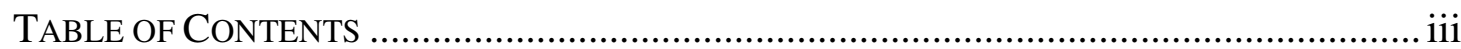

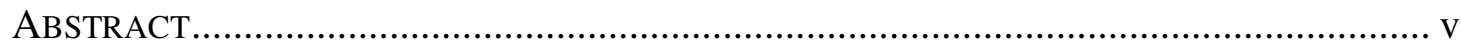

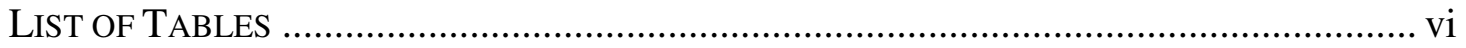

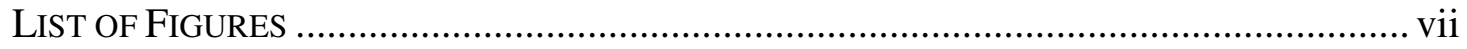

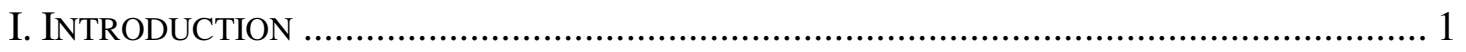

II. BACKGROUND THEORY AND PREVIOUS RESEARCH .............................................. 3

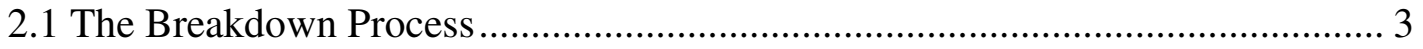

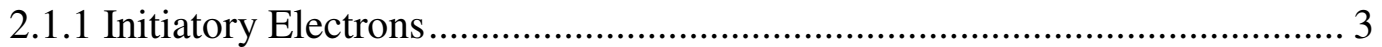

2.1.2 Electron Neutral Collisions ................................................................... 5

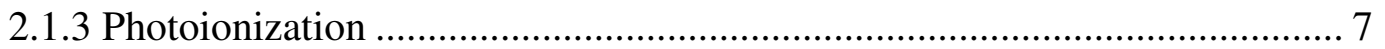

2.1.4 Electron Loss Mechanisms ................................................................... 8

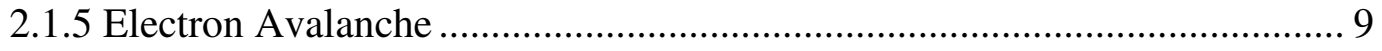

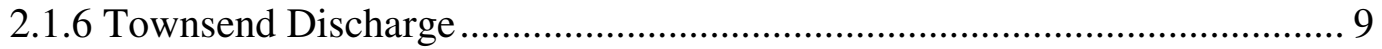

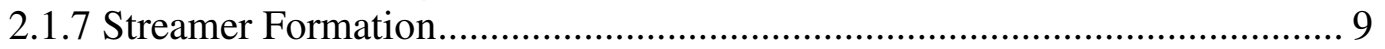

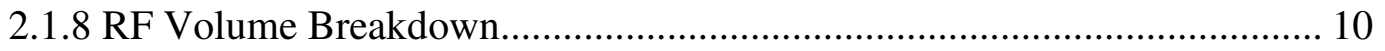

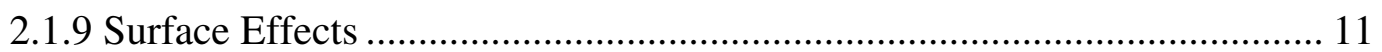

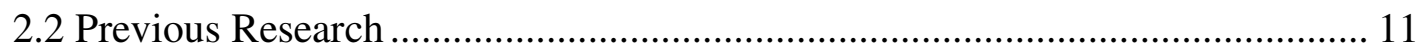

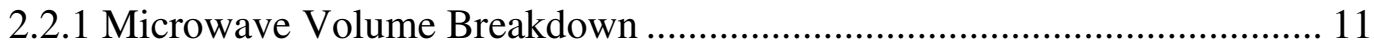

2.2.2 Microwave Surface Flashover................................................................. 12

III. EXPERIMENTAL SETUP........................................................................... 13

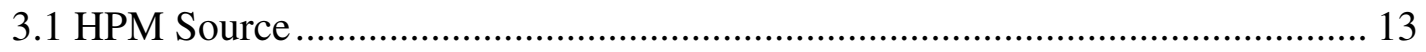

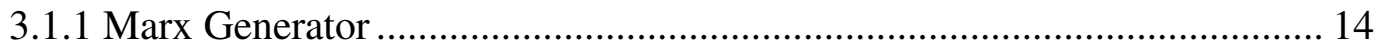

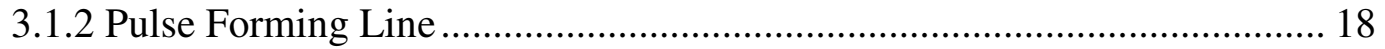

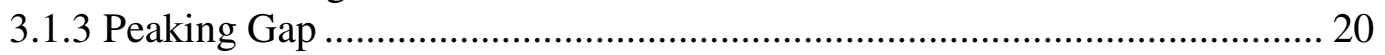

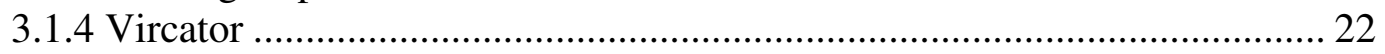

3.2 Circular Waveguide and Breakdown Chamber............................................ 24

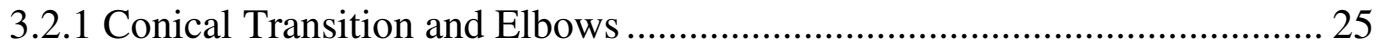

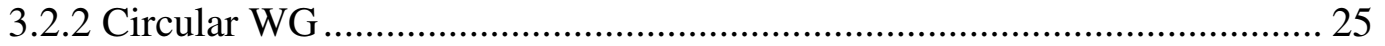

3.2.4 Breakdown Chamber........................................................................... 26

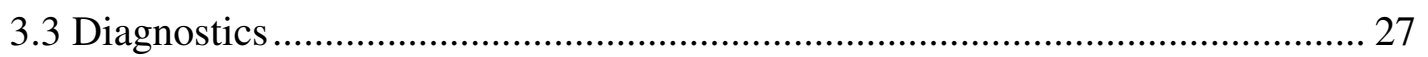

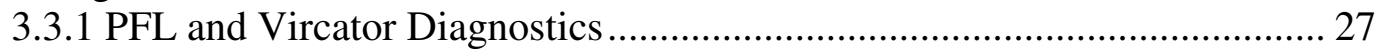

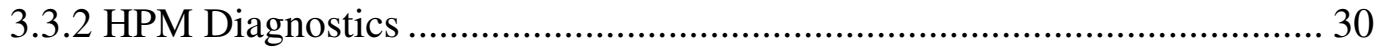

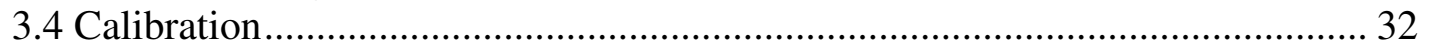

3.4.1 E-Field Probe Calibration ................................................................. 32

3.4.2 WG Power to Flashover E-Field ............................................................ 38

3.4.3 Skewing in a Circular WG .................................................................. 43

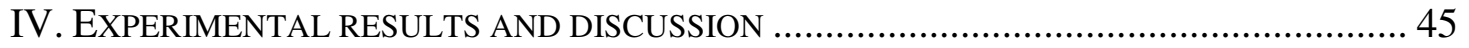

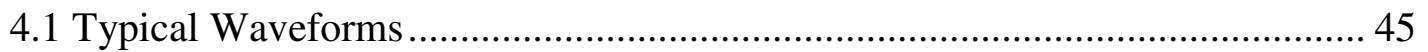

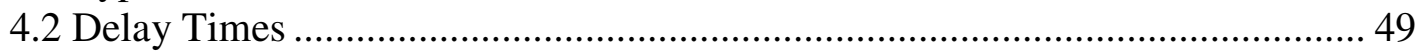

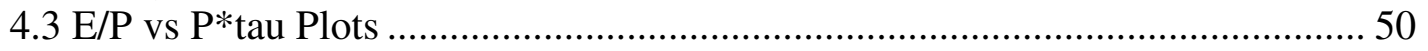

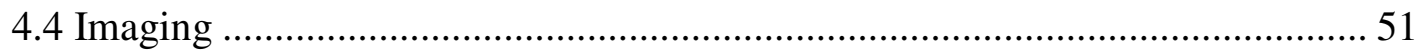

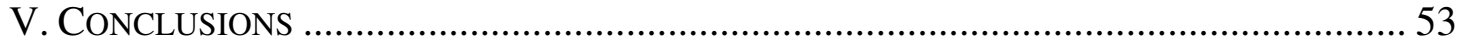




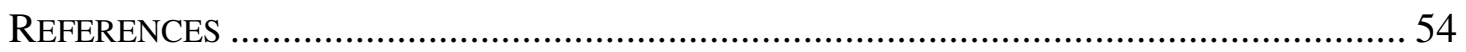

APPENDIX

A: Circular Waveguide Design AND CAlCUlations ............................................ 58

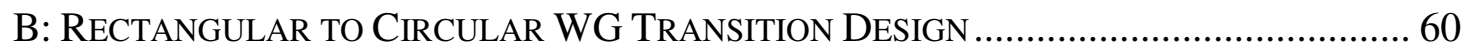




\begin{abstract}
High power microwave (HPM) systems are often limited in transmitted power density and pulse length by surface flashover occurring across dielectric interfaces. This phenomenon can absorb and/or reflect the incident microwave pulse, causing transmission to cease and potentially damaging the microwave source and the dielectric interface itself. The experimental setup is designed to produce and study HPM surface flashover without the influence of a field enhancing triple point. A reflex triode virtual cathode oscillator (vircator) is fed by an 8-stage Marx generator and pulse forming line to produce a $50 \mathrm{MW}, 50 \mathrm{~ns}$ pulse with an adjustable frequency from 3 to $5 \mathrm{GHz}$. This work builds on previous research using a Magnetron generating a $5 \mathrm{MW}, 4 \mu$ s pulse at $2.85 \mathrm{GHz}$, with a flashover setup that is comparable to the current setup, but due to differences in power and pulse length the mechanisms involved have differing impacts on the flashover process. Also under investigation is the influence of factors such as gas pressure and composition. Diagnostics are designed to study the transmitted and incident power levels with sub-nanosecond resolution. Experimental results are discussed and compared with previous testing, literature, and Monte Carlo simulation models.
\end{abstract}




\section{LIST OF TABLES}

2.1: Number of Ion Pairs $n_{i}$ Produced by Cosmic Rays at Different Elevations from Sea Level (per $\left.\mathrm{cm}^{3}-\mathrm{sec}\right)$ where $\mathrm{n}_{\mathrm{i}}=0$ for reference

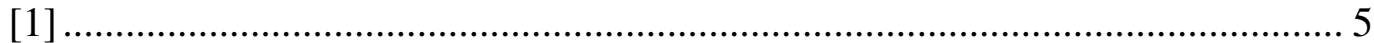

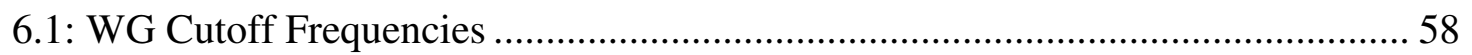

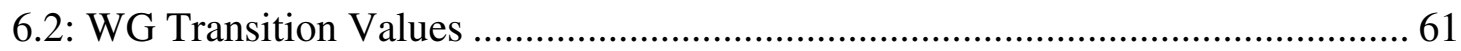




\section{LiST OF FIGURES}

2.1: The lowering of the potential barrier by an external field: curve $1-$ energy curve with no external field, $-\mathrm{W}=\mathrm{e}^{2} / 16 \pi \varepsilon_{0} x$; curve $2-$ energy due to applied field, $-\mathrm{W}_{\mathrm{f}}=\mathrm{eEx}$; curve 3 - total energy

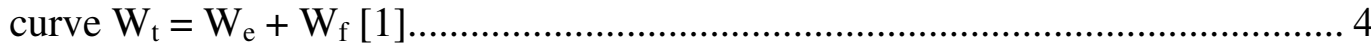

2.2: Total collision cross sections in Argon [11] …………..................................... 7

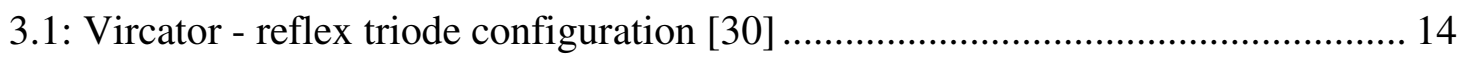

3.2: Ideal Marx generator circuit with load............................................................. 15

3.3: Marx generator circuit with stray capacitances.................................................... 17

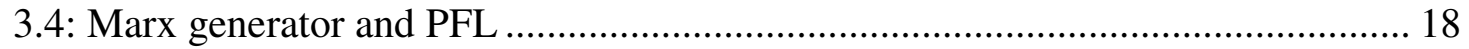

3.5: Marx generator into resistive load [30] ....................................................... 19

3.7: PFL insulator flashover; left figure - Delrin insulator on Marx side of PFL; right figure - Lexan insulator on peaking gap side of PFL .................... 20

3.8: Inner conductor in peaking gap, Lexan insulator, and corona ring....................... 21

3.9: Screen anode and conical vircator insulator after flashover ................................. 23

3.10: Screen anode and cleaned and grooved conical vircator insulator ...................... 23

3.11: Conical transition, circular WG, exit flange, and breakdown

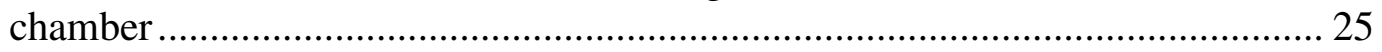

3.12: Vircator, circular WG, and breakdown chamber ............................................ 27

3.13: Capacitive voltage probes ……..................................................................... 28

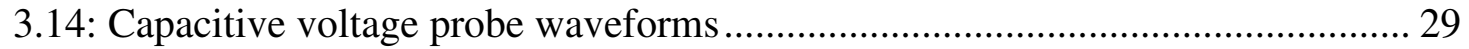

3.15: Rogowski coil waveform with integral ............................................................ 30

3.16: SMA E-field probes with WG field distribution................................................ 31

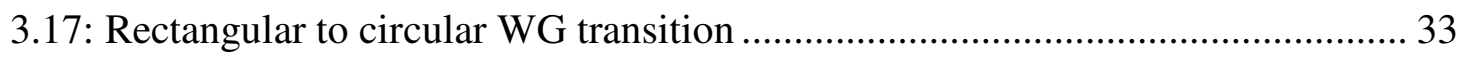

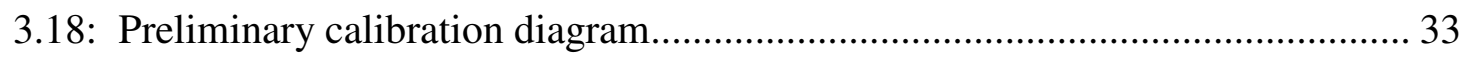

3.19: Final calibration diagram with switch............................................................ 34

3.20: E-probe waveform for calibration with switch ………………………............. 35

3.21: Calibration diagram with directional coupler ................................................... 36

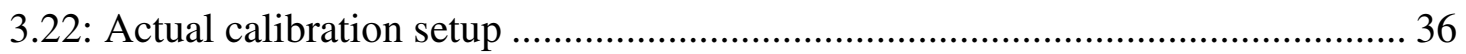

3.23: Directional coupler forward and reverse waveforms ......................................... 37

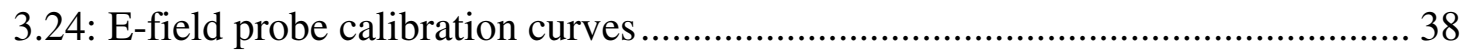

3.25: E-fields inside WG, away from window, excited by $1 \mathrm{~W}$.................................. 39

3.26: E-fields across inside of window, excited by $1 \mathrm{~W}$................................................ 40 
3.27: E-fields across outside of window, excited by $1 \mathrm{~W}$.............................................. 41

3.28: E-Field at WG center along z-axis, excited by $1 \mathrm{~W}$............................................ 42

3.29: Top and side probe voltage magnitude variation with WG angular

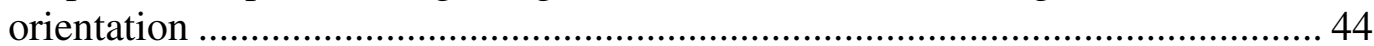

4.1: Typical probe and antenna waveforms, no flashover occurring .......................... 46

4.2: Typical probe and antenna waveforms, flashover at 5 Torr in air ........................ 47

4.2: Typical probe and antenna waveforms of flashover at 5, 50, and 90

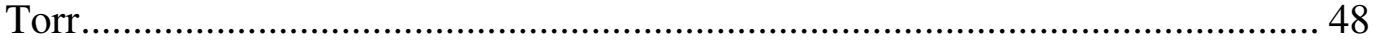

4.3: Delay times from 5 to 90 Torr ………………….......................................... 49

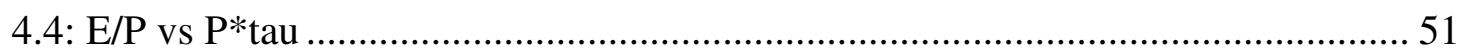

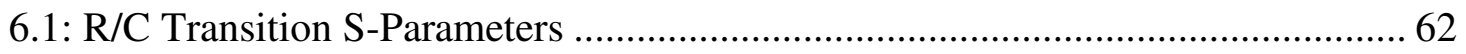




\section{CHAPTER I}

\section{INTRODUCTION}

Electrical breakdown is a phenomenon of considerable importance in many fields of applied physics and engineering. In general, electrical breakdown occurs when a normally insulating medium becomes ionized due to the application of an external electric field and begins to conduct current. In some cases this behavior is desired, as in a spark gap closing a switch, but breakdown often occurs in a way that interrupts device operation, possibly damaging the system. In most high power microwave (HPM) systems, an intense electron beam is used to generate microwaves, which requires a near vacuum environment in order to interact with the structure without undergoing collisions with gas molecules that would interrupt their oscillations. A vacuum is also often required in these systems to prevent internal breakdown due to the high voltages typically involved. In order to transmit these microwaves, a dielectric window is used to separate the vacuum portion of the system from the atmosphere in which the HPM is to be radiated. In some HPM systems, microwaves are generated in vacuum, transmitted through a dielectric interface to a waveguide filled with an insulating medium like sulfur hexafluoride $\left(\mathrm{SF}_{6}\right)$ to inhibit breakdown, and then through another dielectric interface out into the atmosphere. At high electric field (E-field) levels, breakdown can occur on or near one of these dielectric surfaces, in which case it is called surface flashover. Flashover can occur on either side of the window in vacuum, $\mathrm{SF}_{6}$, or at atmospheric pressures, though the focus of this investigation is on flashover in air at low atmospheric pressures, corresponding to high altitudes. Most HPM systems are designed to mitigate the field enhancement found at triple points, which mark the intersection of metal, dielectric, and air, so this experiment is designed to produce surface flashover without a triple point, which would overshadow other mechanisms involved.

When surface flashover occurs in an HPM system, plasma is produced across the window which can absorb and/or reflect the incident microwave pulse. This causes the transmission of microwaves to cease and the reflected pulse may cause damage to 
the HPM source. Repeated flashover events can also impair the structural integrity of the dielectric window, which is already under stress due to the large pressure difference across it, potentially leading to cracking or breaking of the dielectric window. Because of these detrimental effects of HPM surface flashover, it is a major limitation of transmitted power levels and pulse durations in HPM systems. Achieving higher power levels and longer pulse lengths is of considerable interest in several applications of this technology, notably radar, communication, and directed energy systems. In order to diminish this limitation in the development of new HPM systems, better understanding and modeling of this phenomenon and the mechanisms involved are required.

$\mathrm{RF}$ volume breakdown has been widely studied and described in literature [18], but HPM surface flashover has received relatively little attention. Research on surface flashover in vacuum with and without a triple point has been undertaken and the mechanisms involved described [13-19], though these models are significantly altered in the presence of gas molecules. HPM surface flashover at atmospheric pressures without a triple point has recently been investigated using a magnetron producing a $5 \mathrm{MW}, 4 \mu$ s pulse at $2.85 \mathrm{GHz}$ and has shed light on additional processes that play an enhanced role in the presence of a gas and the effects of varying parameters such as gas pressure and temperature [10, 25-29]. Current research builds on this work using a reflex triode virtual cathode oscillator (vircator), which produces a $50 \mathrm{MW}, 50 \mathrm{~ns}$ pulse with an adjustable frequency from 3 to $5 \mathrm{GHz}$. Although there are significant similarities between the two experimental setups, the large differences in power and pulse length cause mechanisms to take on differing degrees of importance.

The following section will give a brief overview of the breakdown process and the mechanisms that contribute to it as well as previous research in this area. This paper will then describe the experimental setup, including diagnostics and representative waveforms. Experimental results and discussion will then be presented, as well as conclusions drawn from this study. 


\section{ChAPTER II}

\section{BACKGROUND THEORY AND PREVIOUS RESEARCH}

In order to gain a better insight into surface flashover, the process itself and the fundamental mechanisms that govern it are identified and described. Electrical breakdown is generally described by a normally insulating medium, composed of neutral molecules, which begins to exhibit conductivity in the presence of an applied electric field due to the production and multiplication of charge carrying electrons and ions. This can occur in solid, liquid, or gaseous media, though the focus of this investigation is on breakdown in gas. Surface flashover is a particular type of electrical breakdown that occurs on or near a dielectric interface. Of critical importance is how free electrons are produced and lost and their behavior and interactions with other particles during the breakdown process. Although some of the processes presented do not play a major role in surface flashover, they do play a role in other types of breakdown encountered in this research, including closing gaps in liquid and gaseous media and the prevention of unwanted breakdown in high field regions. The following section will describe the basic breakdown process and identify significant mechanisms, though a more detailed description can be found in [1-8].

\subsection{The Breakdown Process}

\subsubsection{Initiatory Electrons}

Electrical breakdown generally occurs when initiatory, or seed, electrons are accelerated by an applied electric field such that they gain energy and multiply exponentially. These seed electrons can come from a variety of sources that do not require a preexisting free electron, the most important of which are field emission and cosmic radiation.

Field emission occurs when an electron is liberated from a metallic surface and gains enough energy to overcome the potential barrier due to its "image" charge. At low temperatures, in the absence of an external electric field, the probability of this occurring is small and electrons are emitted sporadically over a long time period. In 
the presence of an external field, the potential barrier is lowered so that less energetic electrons are able to tunnel through the barrier and escape from the surface, as shown in Fig. 2.1. Although the objective of this research into surface flashover is to minimize the effects of a triple point and the enhanced field emission that follows, field emission is encountered in this project at the vircator cathode, spark gap electrodes, and at high field regions along triple points where unwanted flashover can occur. Field emission directly from an insulator can occur, but typically requires very strong electric fields so its effects are often negligible.

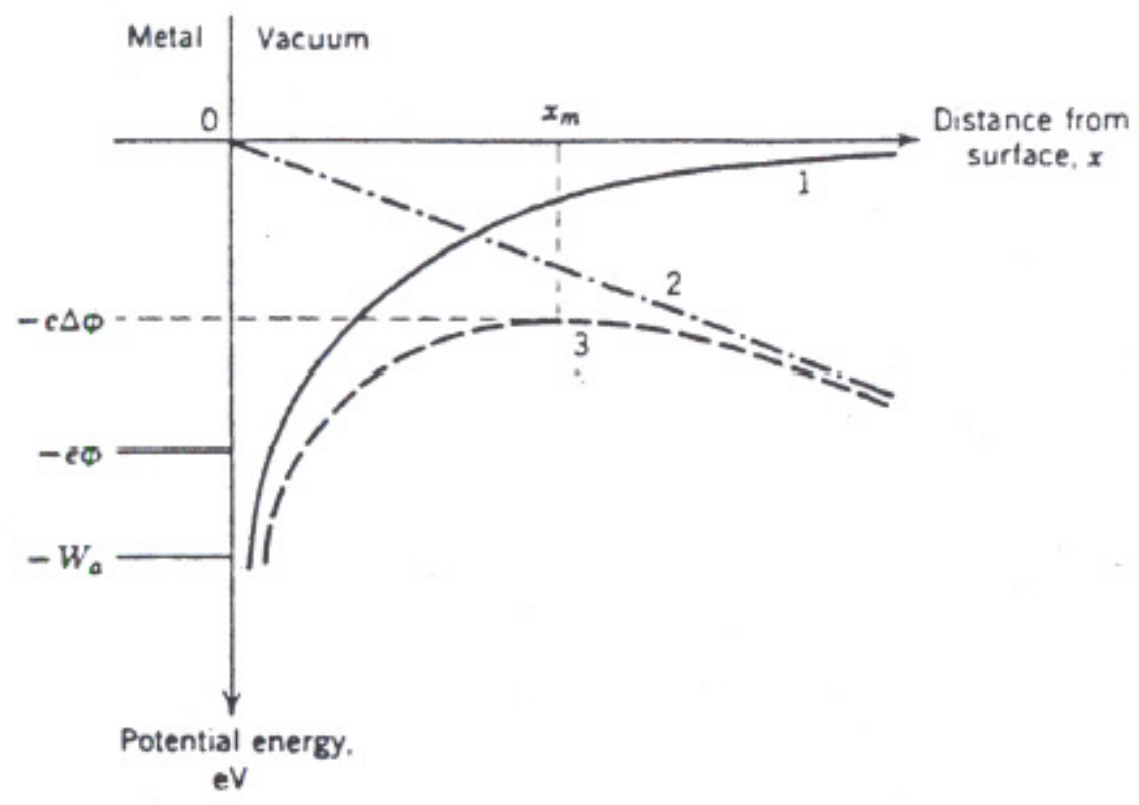

Figure 2.1: The lowering of the potential barrier by an external field: curve 1 - energy curve with no external field, $-\mathrm{W}=\mathrm{e}^{2} / 16 \pi \varepsilon_{0} x$; curve 2 - energy due to applied field, $\mathrm{W}_{\mathrm{f}}=\mathrm{eEx}$; curve 3 - total energy curve $\mathrm{W}_{\mathrm{t}}=\mathrm{W}_{\mathrm{e}}+\mathrm{W}_{\mathrm{f}}[1]$

Cosmic radiation is often the dominant mechanism by which free electrons are generated, especially in the absence of a triple point and field emission. High energy particles originating in outer space continually enter Earth's atmosphere, undergoing collisions that produce free electrons as well as nuclear reactions that can produce secondary cosmic radiation. Because the majority of these particles are protons that can penetrate most types of walls, they can be considered as a constant source of electron-ion pairs in virtually all environments. The intensity of cosmic rays increases 
dramatically with altitude, although the decrease in gas pressure somewhat counteracts the resulting increase in electron production. Table 2.1 gives the relative number of electron-ion pairs produced per $\mathrm{cm}^{3}$ per second from sea level to $9 \mathrm{~km}$. The generally accepted value for production at atmospheric pressure in air is 2-6 pairs $\mathrm{cm}^{-3} \mathrm{~s}^{-1}[8-10]$.

Table 2.1: Number of Ion Pairs $\mathrm{n}_{\mathrm{i}}$ Produced by Cosmic Rays at Different Elevations from Sea Level (per $\mathrm{cm}^{3}-\mathrm{sec}$ ) where $\mathrm{n}_{\mathrm{i}}=0$ for reference [1]

$\begin{array}{lcccccccccc}\text { Elevation } 10^{3} \mathrm{~m} & 0 & 1 & 2 & 3 & 4 & 5 & 6 & 7 & 8 & 9 \\ \mathrm{n}_{\mathrm{i}} \text { ions } / \mathrm{cm}^{3}-\mathrm{sec} & 0 & -1.5 & 1.2 & 4 & 8.3 & 16.5 & 28.7 & 44.2 & 61.3 & 80.4\end{array}$

Terrestrial radiation from the decay of radioactive substances on Earth can also produce initiatory electrons, but the majority of this radiation is composed of $\alpha$ and $\beta$ particles, which cannot penetrate metallic walls and thus play a reduced role in breakdown development in a laboratory setting. Initiatory electrons can also be produced by a host of other processes, including several types of photon and detachment processes, which are discussed in [1, 8-10].

\subsubsection{Electron Neutral Collisions}

In the presence of an external electric field, charged particles experience a drift motion with a drift velocity given by [8]:

$$
v_{d}=\frac{-e}{m \cdot v_{c}}\left(\frac{E_{0}}{1+\frac{\omega^{2}}{v_{c}^{2}}}\right)\left(\cos \omega t+\frac{\omega}{v_{c}} \sin \omega t\right)
$$

Where $\mathrm{e}$ is the charge of the particle, $\mathrm{m}$ is the mass, $v_{\mathrm{c}}$ is the electron-neutral elastic collision frequency, and $\mathrm{E}_{0}$ is the magnitude of the oscillating electric field at an angular frequency of $\omega$. Because this velocity is inversely proportional to the mass of the particle, the much more massive neutral particles and ions accelerate much less than electrons and typically can be considered stationary.

When electrons collide with neutral gas molecules several types of processes can occur, the probabilities of which are highly dependent on the external field and gas pressure, as well as the energy of the colliding electron. When the kinetic energy and momentum of the particles are conserved and no ionization occurs, the collision is 
elastic and can be viewed as an energy loss mechanism. Inelastic collisions occur when some of the kinetic energy of the electron is converted to the potential energy of a gas molecule. An inelastic collision can result in a bound electron achieving a higher energy level (excitation) or the bound electron escaping from the molecule altogether (first-order direct ionization), producing a free electron and a positive ion. This ionization process is of primary interest, but there are many other types of ionizing collisions that can occur and are described in [1-4]. Excitation collisions are sometimes viewed as an energy loss mechanism since no ionization occurs and the electron loses energy, but the increased energy of a bound electron can have additional consequences by reducing the energy required for a later ionization mechanism or the electron can drop to a lower energy level, emitting a photon.

The probability that a particular type of collision will occur can be studied by examining its collision cross section, which is a measure of how close an electron must pass a molecule for the collision to occur. These cross sections are highly dependent on the energy of the electron and some basic collision cross sections for Argon are shown in Fig. 2.2. At low energies, elastic cross sections are higher than inelastic cross sections, but at very low and very high energies, the cross sections of both become very small and collisions less likely. 


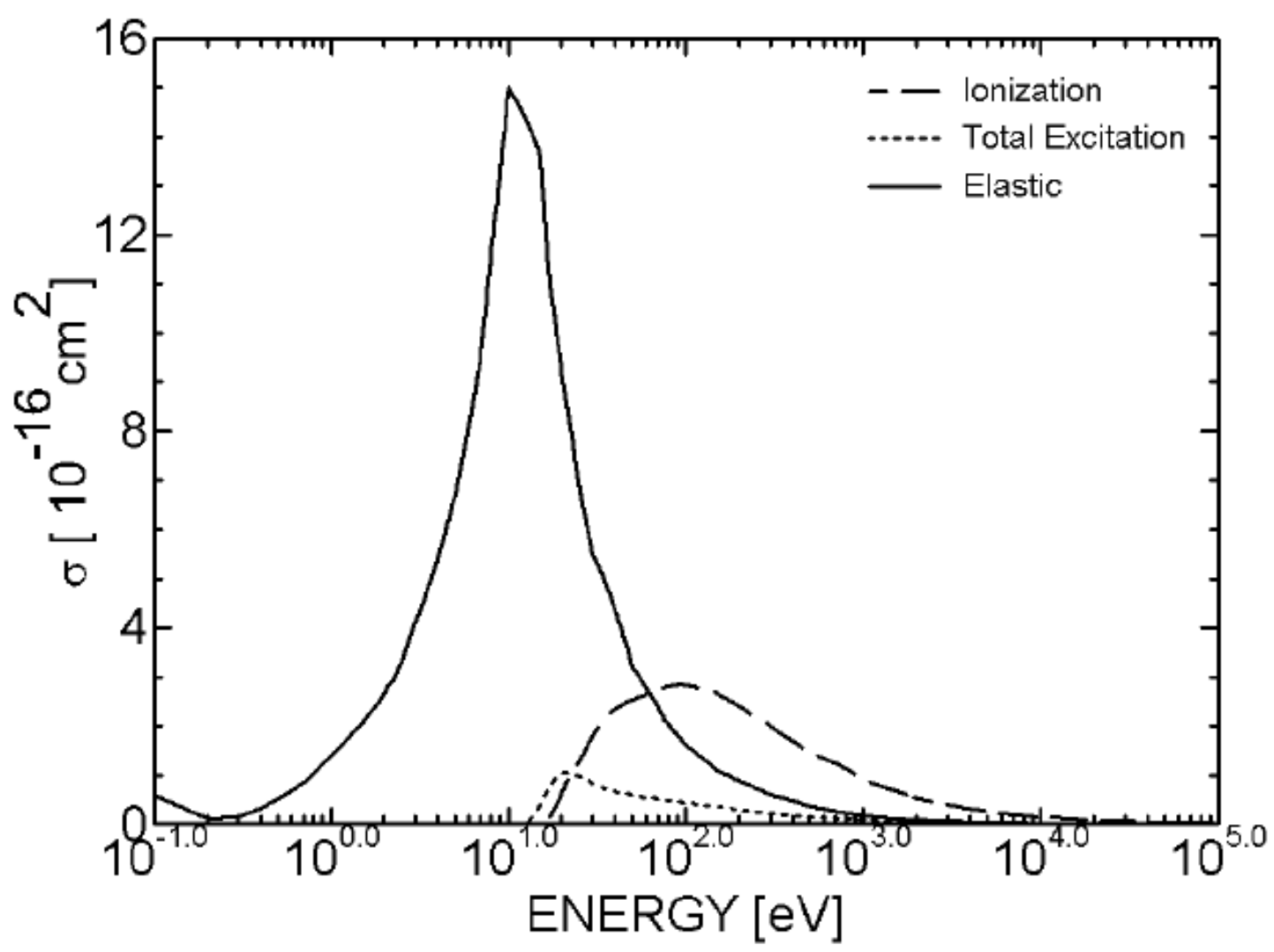

Figure 2.2: Total collision cross sections in Argon [11]

In general, when the field strength is relatively weak electrons gain less energy and are more likely to undergo elastic collisions with gas molecules and lose most of the kinetic energy gained from the external field. Higher fields result in an increase in electron energy, but at very high energies collisions of all types become less likely. At higher pressures, the collision frequency increases and electrons gain less energy between collisions, increasing the likelihood of elastic collisions. At lower pressures, the collision frequency decreases and electrons gain more energy between collisions, increasing the probability of an inelastic collision, but overall collisions become less likely.

\subsubsection{Photoionization}

Ionization can also occur by a bound electron absorbing a photon with enough energy to free it from the molecule, where any excess energy is converted to the 
kinetic energy of the freed electron. When this occurs in a gas it is known as photoionization, while on a surface it is called photoemission. Because the energy of a photon is directly proportional to its frequency, UV and other high frequency sources are more likely to generate photoionization. These photons can come from a variety of sources, including external light as well as photons produced by excited electrons dropping to a lower energy level. Photons can also be released by processes in which excess energy is converted to a photon like recombination or certain types of collisions. When photoemission from a metallic surface occurs, the freed electron is immediately replaced, but when it occurs from a dielectric, the surface builds up a positive charge, which can have significant effects on the behavior of particles near the surface.

\subsubsection{Electron Loss Mechanisms}

Ionization processes result in the multiplication of free electrons, but there are also electron loss mechanisms that inhibit this growth. Attachment occurs when a free electron attaches to a neutral molecule, forming a stable negative ion. Although this particle is still charged, its mass is much larger than that of an electron so it experiences much less acceleration and plays a diminished role in free electron production. Electronegative molecules like $\mathrm{O}_{2}$ and $\mathrm{SF}_{6}$ are much more likely to form negative ions because they have vacancies in their outer shells, so they are often used to inhibit breakdown by scavenging free electrons.

As ionization occurs and the density of electrons grows, electrons move away from the potential discharge channel because of the random motion of the particles in the gas. This mechanism is typically less pronounced than attachment, but their relative importance varies with gas composition and pressure and breakdown dimensions. Recombination occurs when a positive ion recombines with a free electron to produce a neutral particle. This is highly dependent on the density of positive ions available for this reaction and is typically negligible compared to attachment and diffusion. 


\subsubsection{Electron Avalanche}

If conditions are such that ionization processes outweigh electron loss mechanisms then free electrons multiply exponentially, generating a highly dense region of electrons known as an electron avalanche. In a parallel plate geometry with a DC field, the electron density can be modeled by [1]:

$$
n=n_{0} * e^{\left(\alpha^{*} d\right)}
$$

In the above equation, $\mathrm{n}_{0}$ is the initial electron density, $\mathrm{d}$ is the gap spacing, and $\alpha$ is the number of electrons produced per unit length, known as Townsend's first ionization coefficient, and is given by [1]:

$$
\alpha=A p * e^{\left(\frac{-B p}{E}\right)}
$$

Where $\mathrm{A}$ and $\mathrm{B}$ are gas constants found in literature [2], $\mathrm{p}$ is pressure, and $\mathrm{E}$ is the applied electric field.

\subsubsection{Townsend Discharge}

The above treatment of the electron avalanche neglects secondary processes that produce additional avalanches through feedback mechanisms such as photoionization produced by photons released by recombination or excited molecules dropping to lower energy states. These secondary processes generate additional free electrons away from the primary avalanche, which accelerate and produce secondary avalanches. The combined effects of secondary processes are described by Townsend's second ionization coefficient given by [1]:

$$
\gamma^{*}\left(e^{\left(\alpha^{*} d\right)}-1\right)=1
$$

indicating that each seed electron must produce at least one secondary electron to make the discharge self-sustaining.

\subsubsection{Streamer Formation}

When the avalanche reaches a critical size, which occurs when the space charge of the avalanche is comparable to the applied field, the Townsend discharge transitions to streamer formation. Because of the high space charge of the avalanche, electrons at the head of the avalanche gain additional energy, increasing photon emission. This increased emission generates secondary streamers. As positive ions 
reach the anode, the gap distance is effectively decreased, increasing the field across the gap and attracting additional secondary streamers. This continues until the positive ions bridge the gap completely, forming a breakdown path.

\subsubsection{RF Volume Breakdown}

The above treatment is valid for unipolar volume breakdown, but the model differs somewhat in the presence of an oscillating field. Since the direction of the field reverses every half cycle, electrons are alternately accelerated in different directions, reducing the amount of energy gained from the external field. The frequency of the field and the collisional frequency play major roles in determining the extent to which electron motion is restricted, as observed in Eq. 2.1.

In general, if electrons in a plasma are displaced from the uniform distribution of ions, they attempt to return to neutral positions but overshoot and oscillate with a plasma frequency given by [12]:

$$
\omega_{p}=\sqrt{\frac{n_{0} \cdot q_{e}^{2}}{\varepsilon_{0} \cdot m_{e}}}
$$

where $\mathrm{n}_{0}$ is the electron density, $\mathrm{q}_{\mathrm{e}}$ is the charge of an electron, $\varepsilon_{0}$ is the permittivity of free space, and $m_{e}$ is the mass of an electron. This behavior has significant consequences when an RF field is incident on a plasma in that waves with a frequency below the plasma frequency will be reflected from the plasma. For a given frequency, Eq. 2.5 can be rearranged to determine the critical density at which an incident wave will be reflected:

$$
n_{c}=\frac{\omega_{p}{ }^{2} \cdot \varepsilon_{0} \cdot m_{e}}{q_{e}{ }^{2}}
$$

Electron densities above this value will reflect the incident wave, but it should be noted that electron densities below this value will still absorb some energy from the incident wave. For the current experiment operating at $3 \mathrm{GHz}$, the critical electron density was calculated to be $1.12 * 10^{11} \mathrm{~cm}^{-3}$. 


\subsubsection{Surface Effects}

Defining the role of a dielectric surface as an electron gain or loss mechanism is complicated by the presence of multiple mechanisms involved, which are dependent on a range of parameters. Secondary electron emission occurs when a free electron strikes the surface, liberating a bound electron by transferring some of its kinetic energy. This process is highly dependent on the energy of the primary electron, as low energies are insufficient to free electrons, and at high energies the primary electron penetrates deeper into the surface and is less likely to diffuse back into the volume.

Photoemission from the surface can also play a role in flashover formation, especially if an external radiation source is present. This mechanism causes additional electrons to be liberated from the surface, but this also causes the surface to develop a positive charge, attracting additional electrons to the surface capable of producing secondary electron emission. Surface defects can also have an impact on the breakdown process, as these sites are more likely to generate free electrons, though the study of these defects is somewhat difficult. At low pressures, gas can be desorbed from the dielectric surface, increasing the local pressure across the dielectric, which has a significant impact on the breakdown process. The following section will describe the previous research that pertains to this investigation.

\subsection{Previous Research}

\subsubsection{Microwave Volume Breakdown}

Significant experimental data and models have been produced over the last half century, comprising a large range of electric fields, pressures, and pulse lengths. A summary of major breakdown studies can be found in [10]. Most of the early work in this field utilized resonant cavity structures to generate large electric fields. Much of this work also used an initiatory electron source such as UV radiation or a radioactive substance to ensure an ample supply of seed electrons such that breakdown behavior was less dependent on the statistical variation in seed electrons. This work has done much to advance the theory of microwave breakdown, though the introduction of a dielectric surface introduces additional mechanisms to the process that deserve further 
study. Also, much of the experimental data was taken at E/p values considerably less than those encountered in the present experiment, which makes comparisons somewhat more difficult. A notable exception to this is the work done by Felsenthal \& Proud [7], which extends to higher E/P values, but was not done under microwave excitation, so the comparison is still not straightforward.

\subsubsection{Microwave Surface Flashover}

Studies of microwave surface flashover have been undertaken by several researchers, though the range of data is more limited than that for volume breakdown and the roles of mechanisms in different regimes is still somewhat nebulous. Investigations into surface flashover at pressures near vacuum [13-19] have described the process by secondary electron emission avalanche (SEEA), which is a combination of processes comprising secondary electron emission, multipactor discharge, electron induced gas desorption, and Paschen breakdown in the desorbed gas. At higher pressures, the effects of secondary electron emission are diminished and the process begins to resemble RF volume breakdown.

Experimental work and simulations of surface flashover at atmospheric pressures has been undertaken at Texas Tech by Calico [20], Krile [21-24], and Edmiston [10, 25-29]. This work has shed valuable insight into the flashover process at atmospheric pressures, though they encompass a wide variety of experimental setups, some with electron injecting triple points. Current work is aimed at expanding upon this research by investigating surface flashover without a triple point at lower pressures and higher field amplitudes in order to gain a better insight into the vaguely defined regime between breakdown in vacuum and at atmospheric pressures. 


\section{CHAPTER III}

\section{EXPERIMENTAL SETUP}

An experimental setup was designed to produce HPM induced dielectric surface flashover, without the influence of a triple point. The HPM produced by the source is transmitted to a dielectric window inside an environmentally controlled chamber so that variables of interest can be controlled and their effects on flashover investigated. Diagnostics are employed to monitor the HPM source operation and to measure microwave power inside the WG and that transmitted past the window. The individual system components will be described more in depth in this chapter.

\subsection{HPM Source}

The HPM source used in this experiment is an experimental reflex triode vircator developed at Texas Tech University by Lara [30] and expanded on by Chen [31-32]. The basic operating principle behind a vircator is that a high voltage pulse is applied across a planar cathode and a screen anode such that electrons are field emitted from the cathode. Since the anode is a screen and mostly transparent, most of the electrons accelerate toward the screen but then pass through it. When the current exceeds the space charge limit, given by the Child-Langmuir relation, a virtual cathode forms on the other side of the anode and the electrons enter an oscillating state, generating microwaves. The frequency of these oscillations is highly dependent on the anode/cathode (AK) gap spacing, so the frequency of the vircator can be controlled by adjusting the gap spacing. Fig. 3.1 is a drawing of the vircator in reflex triode configuration. 


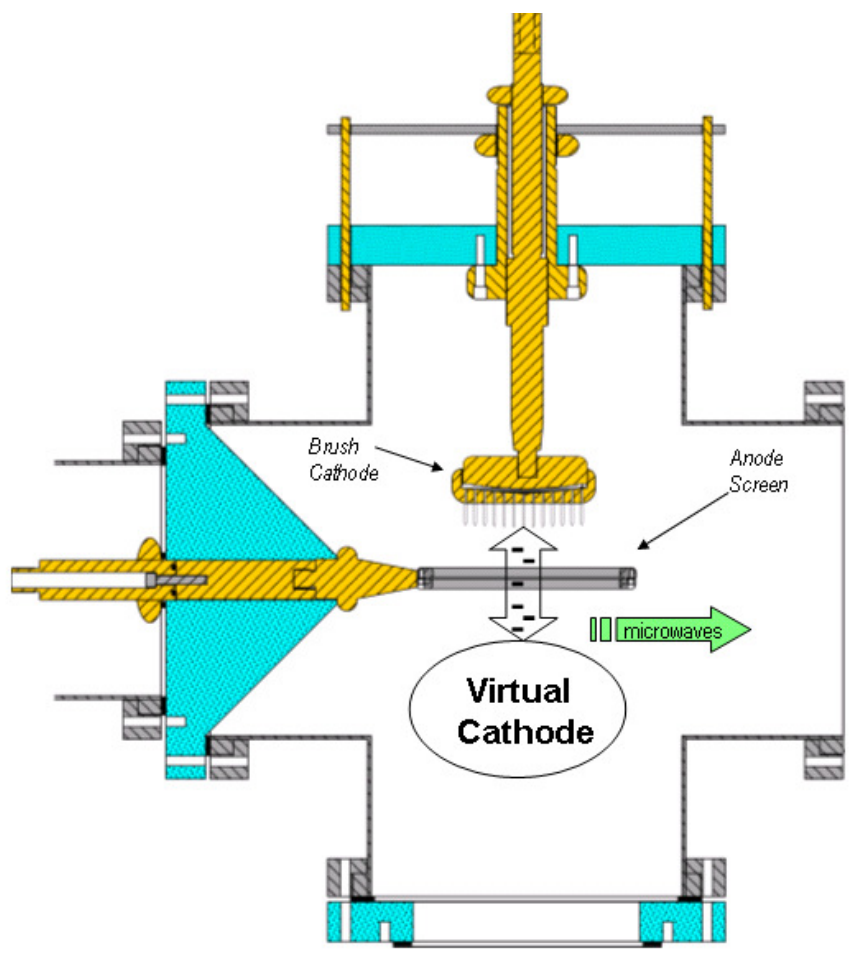

Figure 3.1: Vircator - reflex triode configuration [30]

\subsubsection{Marx Generator}

Since vircators require a high voltage pulse that is typically unavailable in commercial power supplies, voltage multiplication schemes are regularly used to store energy over a long period of time and discharge that energy in a much shorter period of time with much higher power levels. Marx Generators are often used for this purpose, usually because of their relatively simple design and construction. A Marx Generator operates by charging several capacitors in parallel over a relatively long period of time and then momentarily switching them into a series configuration, known as erecting the Marx. This causes the voltage on each capacitor to be summed and discharged into a load over a much shorter period of time, effectively multiplying the charging voltage by the number of capacitors. An ideal schematic of a Marx generator circuit connected to the load is shown in Fig. 3.2. 


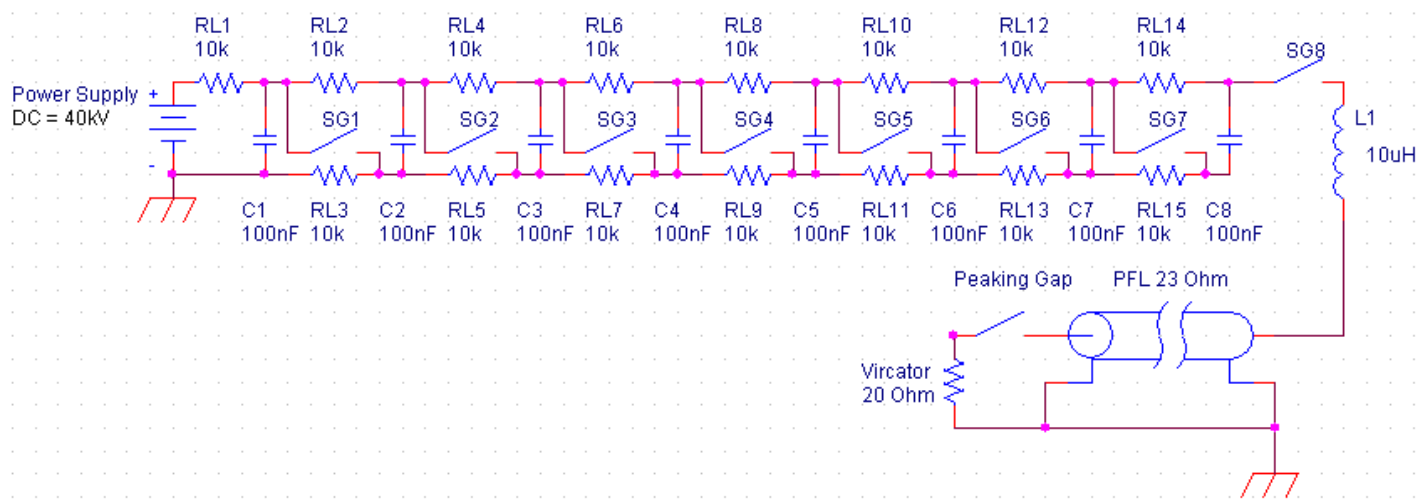

Figure 3.2: Ideal Marx generator circuit with load

The capacitors used for the Marx bank are Maxwell $31885100 \mathrm{nF}$ with a maximum voltage rating of $75 \mathrm{kV}$. Under normal operation the charging voltage is 40 to $45 \mathrm{kV}$, resulting in an erected voltage of 320 to $360 \mathrm{kV}$. Charging and discharging of the Marx is accomplished with an analog Hippotronics HV DC power supply, which provides control and observation of charging voltage and current as well as spark gap pressure, which is manipulated to erect the Marx. The charging current is kept under $6 \mathrm{~mA}$ and typical charging times are on the order of several seconds so the system is effectively single shot. Because large charging resistors isolate the power supply from the capacitors, the voltage measured at the power supply is not necessarily the voltage across the Marx, so charging is accomplished by raising the voltage slightly above the desired charging voltage then allowing the current to reach zero and the voltage to reach the desired level before discharging.

Charging of the Marx in parallel requires the use of high voltage resistors between each stage as depicted in Fig. 3.2. These resistors must be capable of handling high charging voltages and must also have an impedance high enough to appear as an open circuit when the Marx is erected, in this case 5 to $10 \mathrm{k} \Omega$, allowing the voltages on each capacitor to add in series. Several types of resistors could be used to accomplish this, but water/cupric-sulfate resistors were used because a smaller size is required to achieve the same voltage handing as compared with other bulk materials.

Spark gaps are used to switch and erect the Marx, which are filled with air from the building's compressed air line and are held at a pressure of $\sim 30$ psi to prevent 
self breakdown of the gaps during charging. This pressure was found empirically by increasing the pressure in the gaps until self breakdown no longer occurred during charging. Using bottled nitrogen or air helps to improve the consistency of the system, though the advantage gained was not observed to be significant, possibly due to the presence of inconsistencies arising from other components of the system. Another advantage of using bottled gas is that water occasionally finds its way into the compressed air line, which causes the spark gaps to breakdown at a lower voltage and prevents complete charging of the system, in which case the spark gaps must be disassembled and cleaned. The Marx is triggered through the power supply unit by purging the gas line feeding spark gaps 1 and 2 so that the pressure quickly drops and breakdown occurs, causing subsequent stages to breakdown as well. The gaps are arranged such that photons generated by the breakdown of one gap are obstructed from reaching the next gap and helping to initiate breakdown there, as opposed to the more common parallel configuration in which UV radiation produced by one gap helps to break down the next.

The circuit in Fig. 3.2 neglects stray capacitances, which can have a considerable impact on Marx generator operation. Stray capacitances between stage capacitors and ground $(\mathrm{Cs})$ and across switches $(\mathrm{Cg})$ are shown in Fig. 3.3. When the first spark gap is triggered, the charged stage capacitor (C0) is connected in parallel to $\mathrm{Cs} 1$ and charges it to a value nearly equal to the charging voltage, as long as $\mathrm{C} 0$ is much larger than Cs. This causes the voltage across $\mathrm{Cg} 2$ and $\mathrm{Cs} 2$ to be double the charging voltage. These capacitances form a capacitive voltage divider such that the voltage across the second switch is inversely proportional to $1+\mathrm{Cg} / \mathrm{Cs}$ [33]. In order to maximize the voltage across the spark gap and cause it to trigger as fast as possible, Cs must be much larger than Cg. To accomplish this, 1" x 4" copper tabs facing the ground plane were attached to the capacitor connections to increase the stage to ground capacitance. 


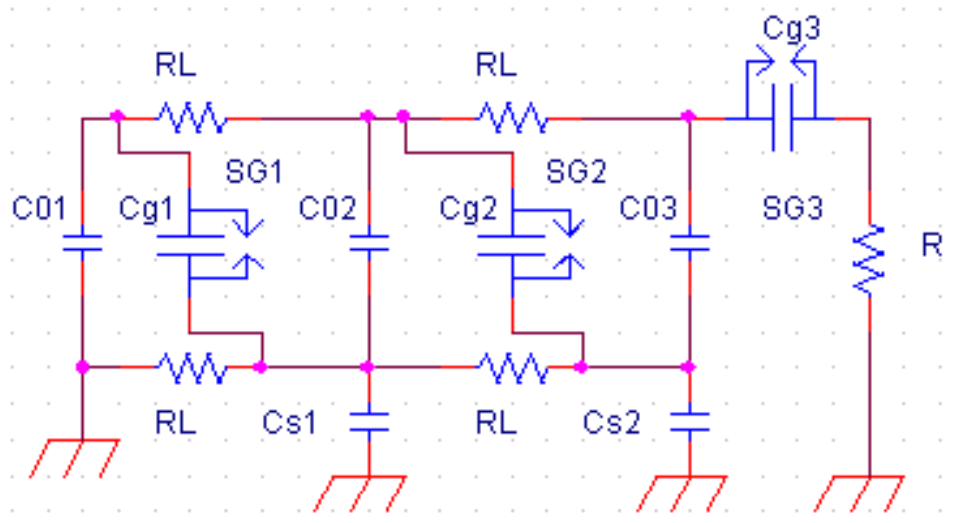

Figure 3.3: Marx generator circuit with stray capacitances

At the physical dimensions involved, the voltages produced by the Marx generator would easily break down air and arc to surrounding structures, bypassing the load and possibly damaging the system, so the entire system of capacitors and spark gaps are submerged in transformer oil. The transformer oil could be eliminated at the cost of much larger system dimensions and/or the use of some other insulating medium. Fig. 3.4 is a picture of the power supply, Marx generator, and the connecting pulse forming line (PFL). 


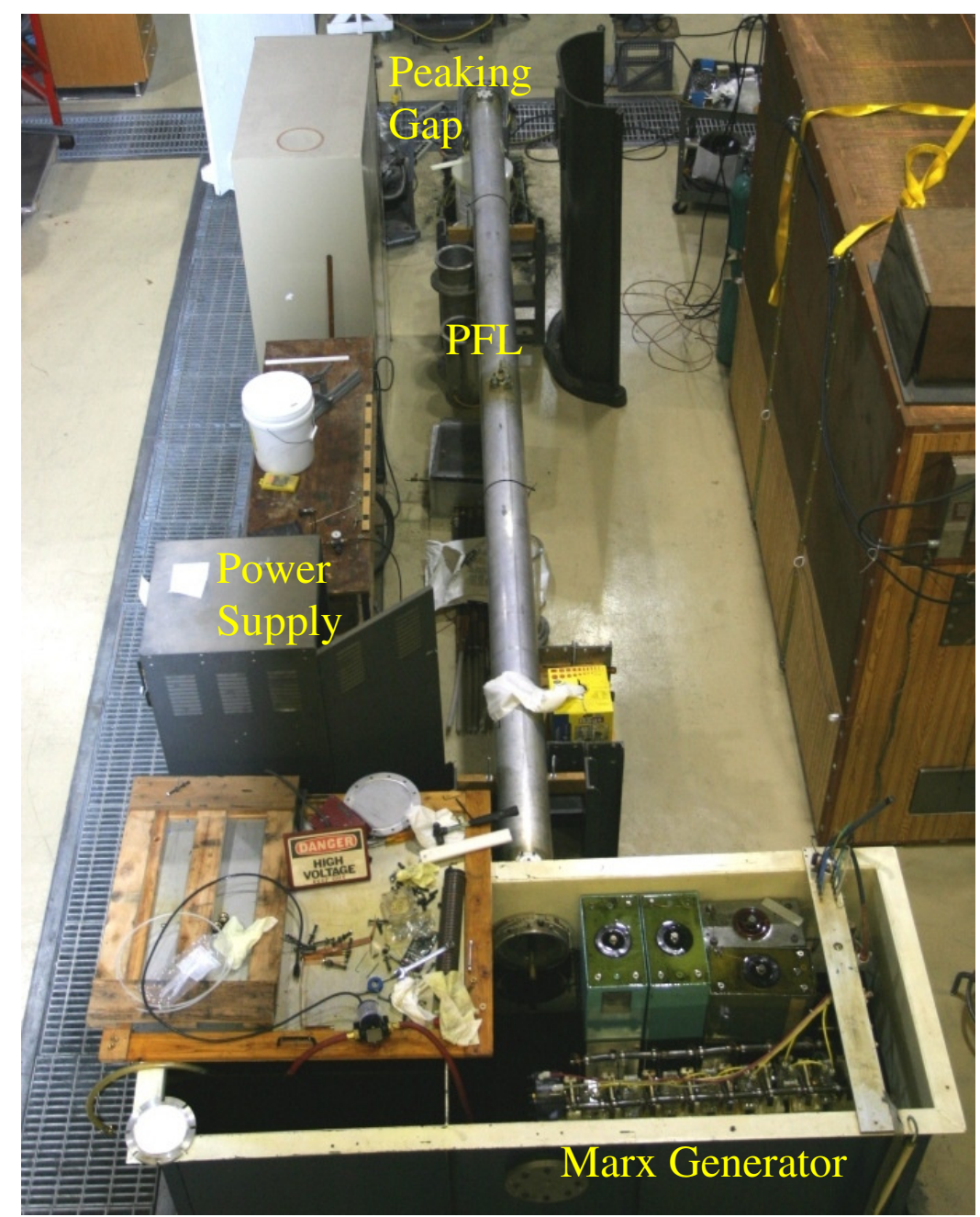

Figure 3.4: Marx generator and PFL

\subsubsection{Pulse Forming Line}

Discharging the Marx generator into a resistive load typically produces a pulse with a width of greater than $200 \mathrm{~ns}$, a risetime of $20 \mathrm{~ns}$, and a nonideal pulse shape due to reflections and stray inductance and capacitance. This behavior can be seen in Fig. 3.5, which was taken by discharging the Marx into a 1200:1 resistive divider. A pulse forming line (PFL) is used to shape the pulse and decrease the pulse length to $60 \mathrm{~ns}$ to reduce the likelihood of breakdown occurring across the vircator, which can become a problem with longer pulse lengths. 


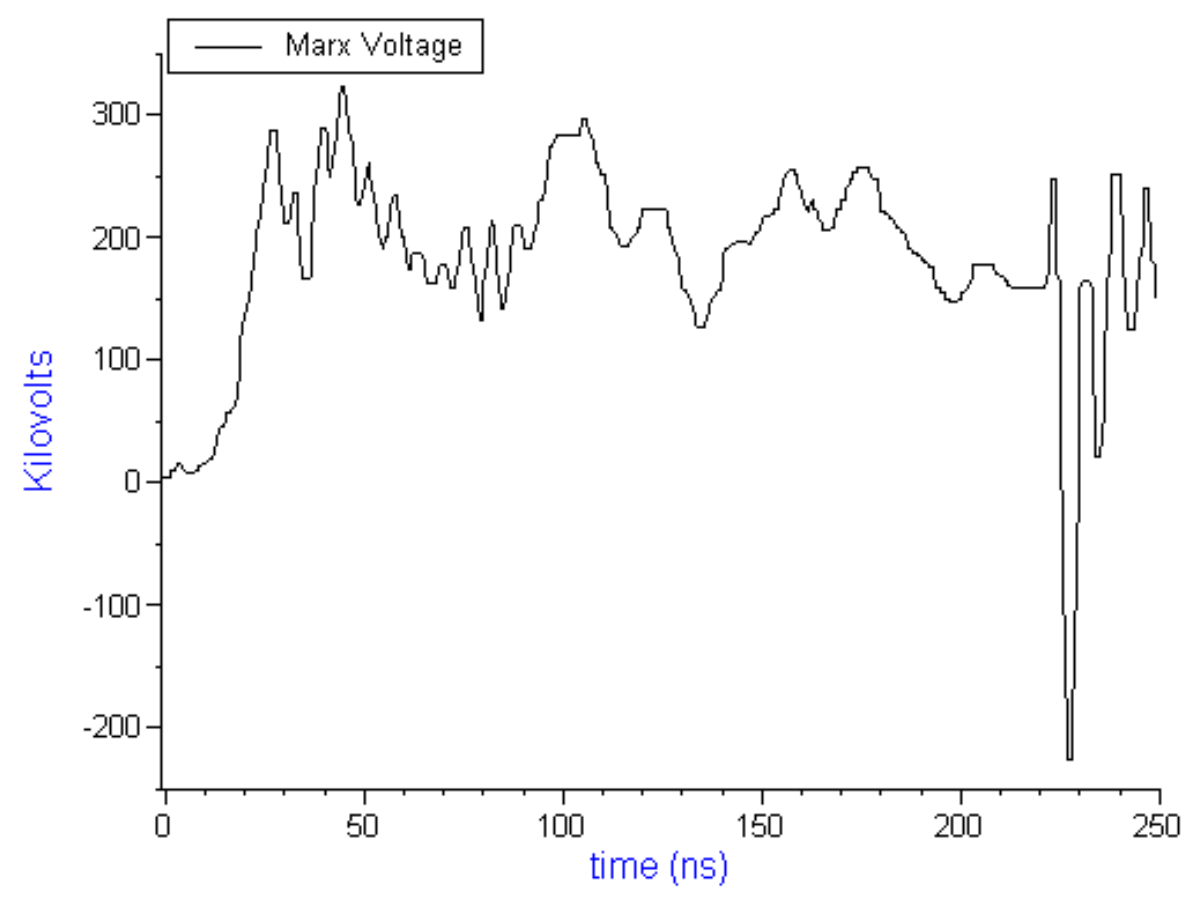

Figure 3.5: Marx generator into resistive load [30]

The PFL is designed to have a $20 \mathrm{Ohm}$ impedance to match the diode impedance of the vircator, a $60 \mathrm{~ns}$ two-way transit time, and to be able to hold-off up to $800 \mathrm{kV}$. The inner conductor is constructed from 4" diameter solid copper tubing and 8 " diameter aluminum tubing is used for the outer conductor. The overall physical length of the PFL is $6 \mathrm{~m}$, yielding a pulse length of $60 \mathrm{~ns}$. The area between conductors is also filled with transformer oil to prevent breakdown from the inner conductor to the outer. Ports are located in the middle and at each end so that trapped air can be removed when filling the line with oil. The inner conductor is supported at both ends by insulators and by a piece of nylon all thread in the middle to prevent sagging. After extensive use, flashover began to occur across the supporting insulators. Fig. 3.6 shows the Delrin insulator on the Marx side of the PFL and the Lexan insulator on the peaking gap side of the PFL after repeated flashover had occurred. 


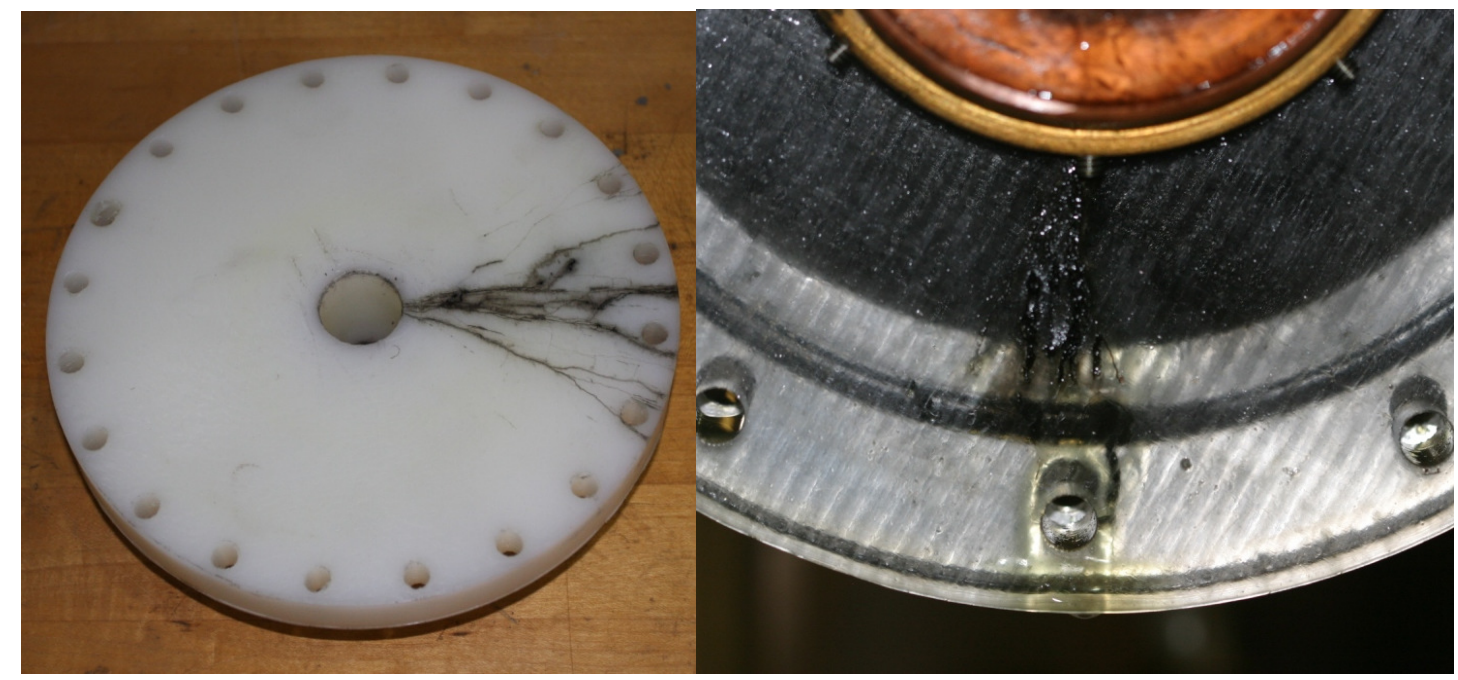

Figure 3.7: PFL insulator flashover; left figure - Delrin insulator on Marx side of PFL; right figure - Lexan insulator on peaking gap side of PFL

This flashover did not inhibit vircator operation as it took longer to develop than the pulse length of the HPM, but it was still undesired as it could lead to structural failure of the insulator or to flashover developing fast enough to inhibit HPM generation. Because the flashover punched through to the other side of the Delrin insulator, a new insulator had to be machined to replace it. The tracking on the Lexan insulator was more shallow so it could be removed by sanding off 1/4". Grooves were then cut on both insulators to inhibit further flashover, which can be seen on the Lexan insulator in Fig. 3.8.

\subsubsection{Peaking Gap}

A peaking gap is used to decrease the risetime of the pulse, which is relatively large due to stray inductance and the inductance of the PFL itself. It is constructed of 1" copper-tungsten electrodes and housed in a separate 8" aluminum chamber, also filled with transformer oil. Because the inner conductor is not attached to the insulating supports and the connection from the Marx bank is not rigid, breakdown in the peaking gap can push the inner conductor away from the peaking gap over multiple firings, effectively increasing the peaking gap spacing and causing the gap to close late or not at all. This increase in gap spacing likely contributed to flashover across the Lexan insulator shown in Fig. 3.7. Brass corona rings are attached to the 
inner conductor on both sides of the dielectric with set screws, as shown in Fig. 3.8. These rounded rings minimize field enhancement at the triple point, inhibiting flashover across the dielectric. They also prevent the inner conductor from moving and altering the peaking gap spacing.

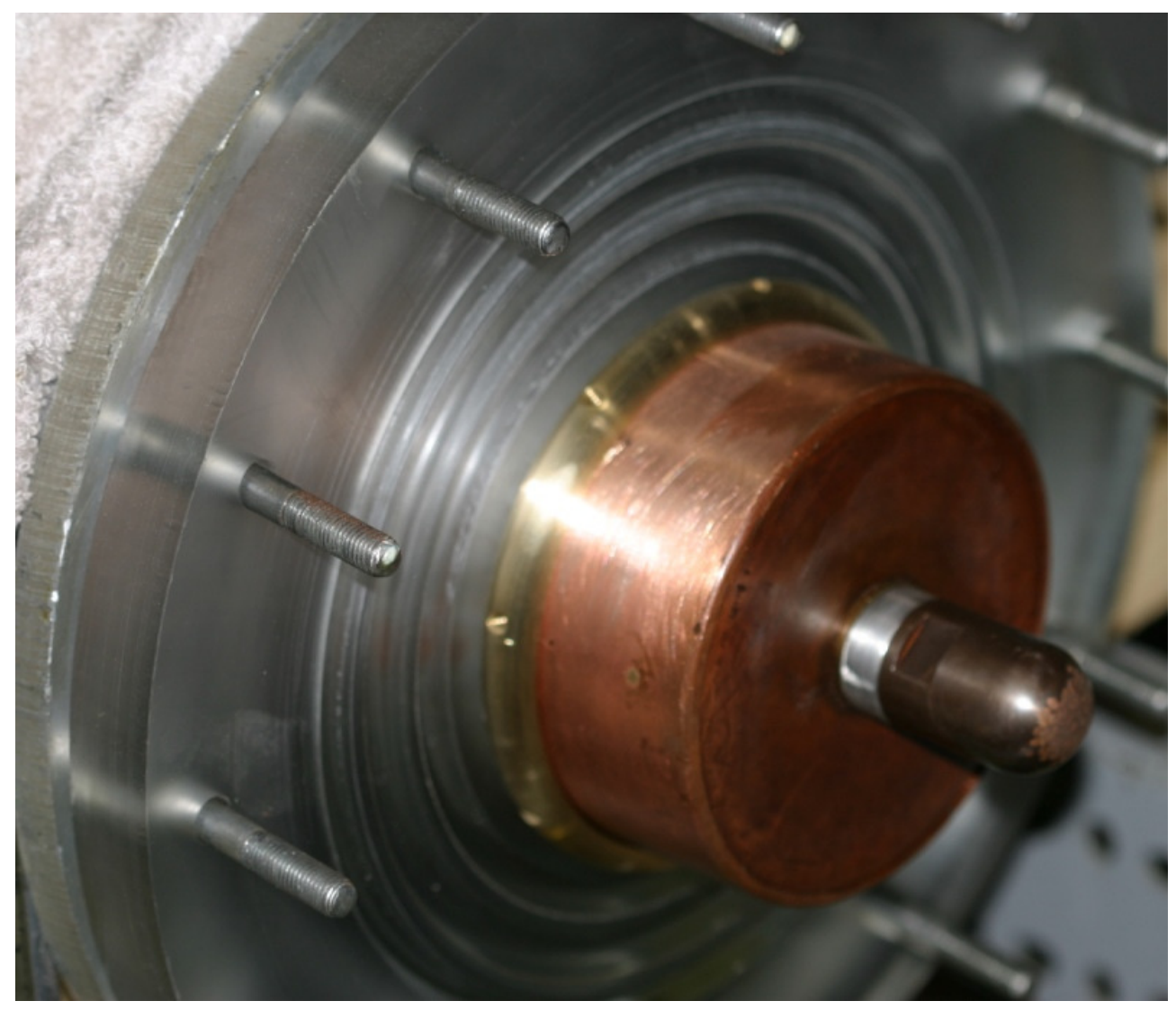

Figure 3.8: Inner conductor in peaking gap, Lexan insulator, and corona ring

In an ideal case, the peaking gap breaks down when the voltage on the transmission line reaches a maximum, delivering to the vircator a $60 \mathrm{~ns}, 360 \mathrm{kV}$ pulse with a risetime on the order of a few ns. The gap spacing was determined by trial and error, though it is somewhat cumbersome to adjust the spacing and requires dismantling the peaking gap to adjust the corona ring. The peaking gap is equipped with ports for the addition and removal of transformer oil from the system. Breakdown in oil produces carbon byproducts that cause subsequent breakdown to develop more quickly, possibly to the detriment of the transmitted pulse voltage, so the oil in the peaking gap is periodically cycled and filtered. The voltages on the transmission line 
before and after the peaking gap are monitored through capacitive voltage probes, which will be further discussed in section 3.3.

\subsubsection{Vircator}

The vircator is housed in an 8" diameter stainless steel chamber attached to the peaking gap chamber. The screen anode of the vircator is connected to the peaking gap electrode opposite the PFL. The screen anode is made out of stainless steel mesh and typical transparency levels are 50-70\%. Higher transparency levels yield higher microwave output, but screens with higher transparencies are more susceptible to melting. A high voltage, conical feed through constructed of Delrin is used to separate the oil filled peaking gap from the vircator under vacuum. The feed through is designed to inhibit breakdown from the feedthrough and screen to the chamber by reducing field enhancement and increasing the distance from the screen to the chamber with a conical shape and grooves [31]. This design has not been completely successful and over time flashover began to occur, shortening the pulse length of the HPM generated. Fig. 3.9 depicts the conical insulator after several flashover events, as indicated by the dark coloring around the top of the cone and tracking across the surface. The insulator was then sanded down and new grooves were cut as shown in Fig. 3.10. 


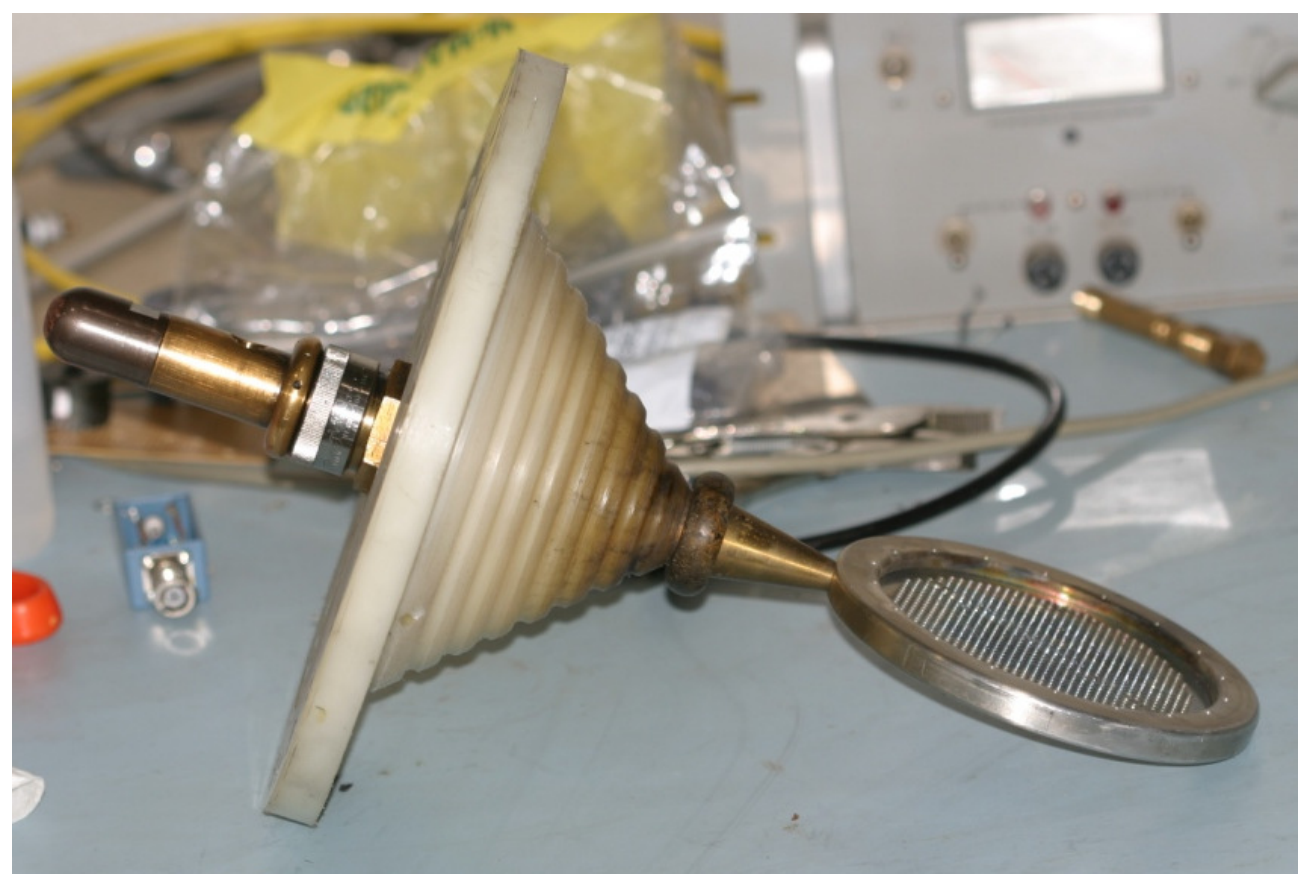

Fig. 3.9: Screen anode and conical vircator insulator after flashover

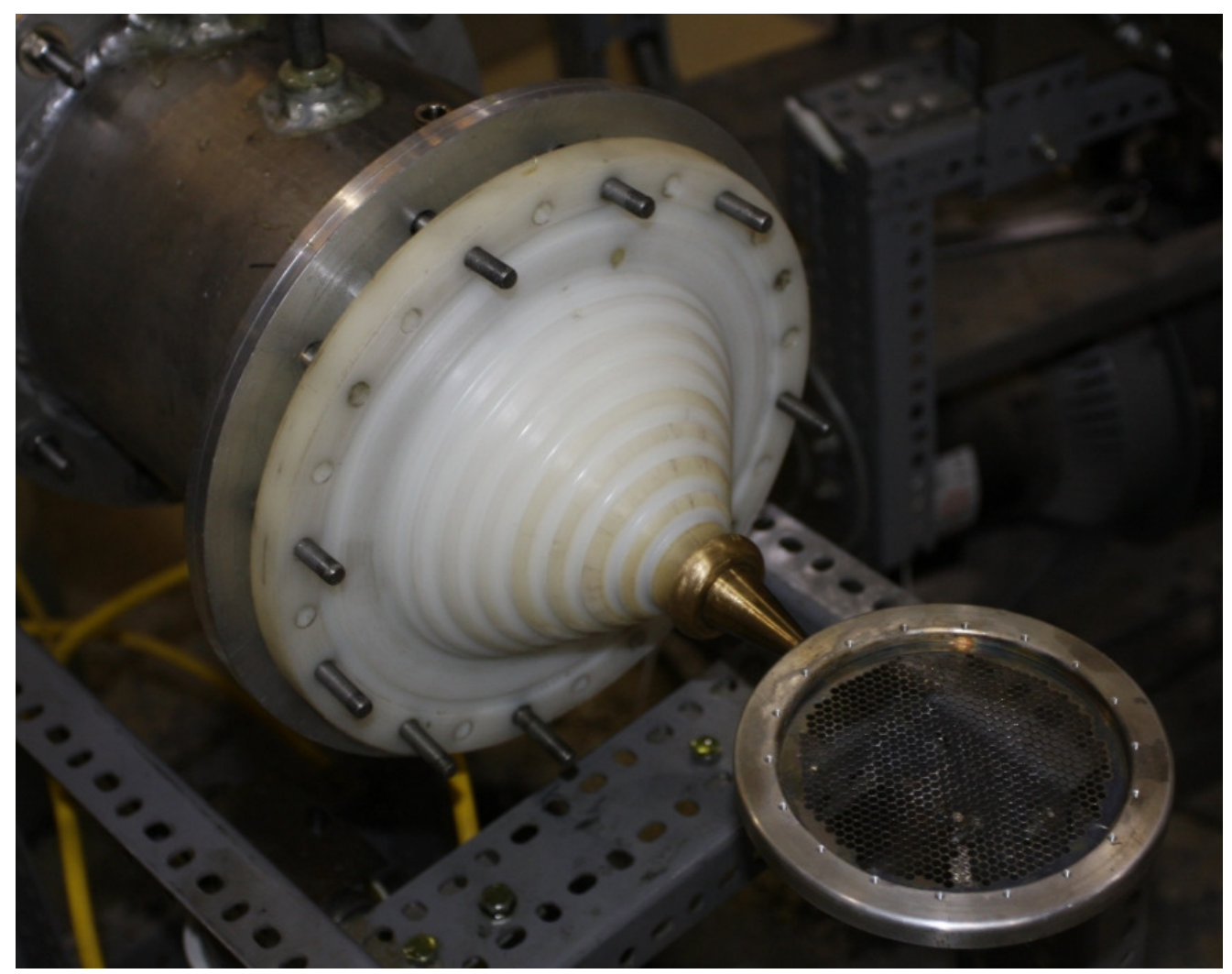

Figure 3.10: Screen anode and cleaned and grooved conical vircator insulator 
A velvet covered brass cathode is positioned above the screen anode as depicted in Fig. 3.1. There are some drawbacks to using a velvet cathode, including additional outgassing and deterioration over time. Testing by Chen [31] demonstrated that comparable results are achieved with microetched aluminum cathodes, which don't experience as much outgassing and generally have a much longer lifetime, but they are also more expensive and difficult to fabricate with little advantage as far as microwave output is concerned. The cathode is held in place by a threaded brass bolt, which allows the AK gap spacing to be adjusted without dismantling the chamber or losing vacuum. The chamber is evacuated to a pressure typically on the order of tens of microTorr using a roughing pump and Varian Turbo-V 500 MacroTorr molecular pump. A Rogowski coil on the cathode bolt allows measurement of the cathode current and will be further discussed in section 3.3.

\subsection{Circular Waveguide and Breakdown Chamber}

The HPM generated by the vircator passes through a Lexan dielectric window and through an aluminum circular WG to the dielectric window and breakdown chamber as shown in Fig. 3.11. The diameter of the WG was chosen to be 2.6" in order to achieve a dominant $\mathrm{TE}_{11}$ mode cutoff frequency of $2.6 \mathrm{GHz}$ and a next higher order $\mathrm{TM}_{01}$ mode cutoff frequency of $3.4 \mathrm{GHz}$, ensuring single dominant mode operation in that frequency range (see Appendix A). The AK spacing was adjusted by trial and error to produce a frequency of $3 \mathrm{GHz}$ and typically varied by less than 0.2 $\mathrm{GHz}$ from shot to shot. 


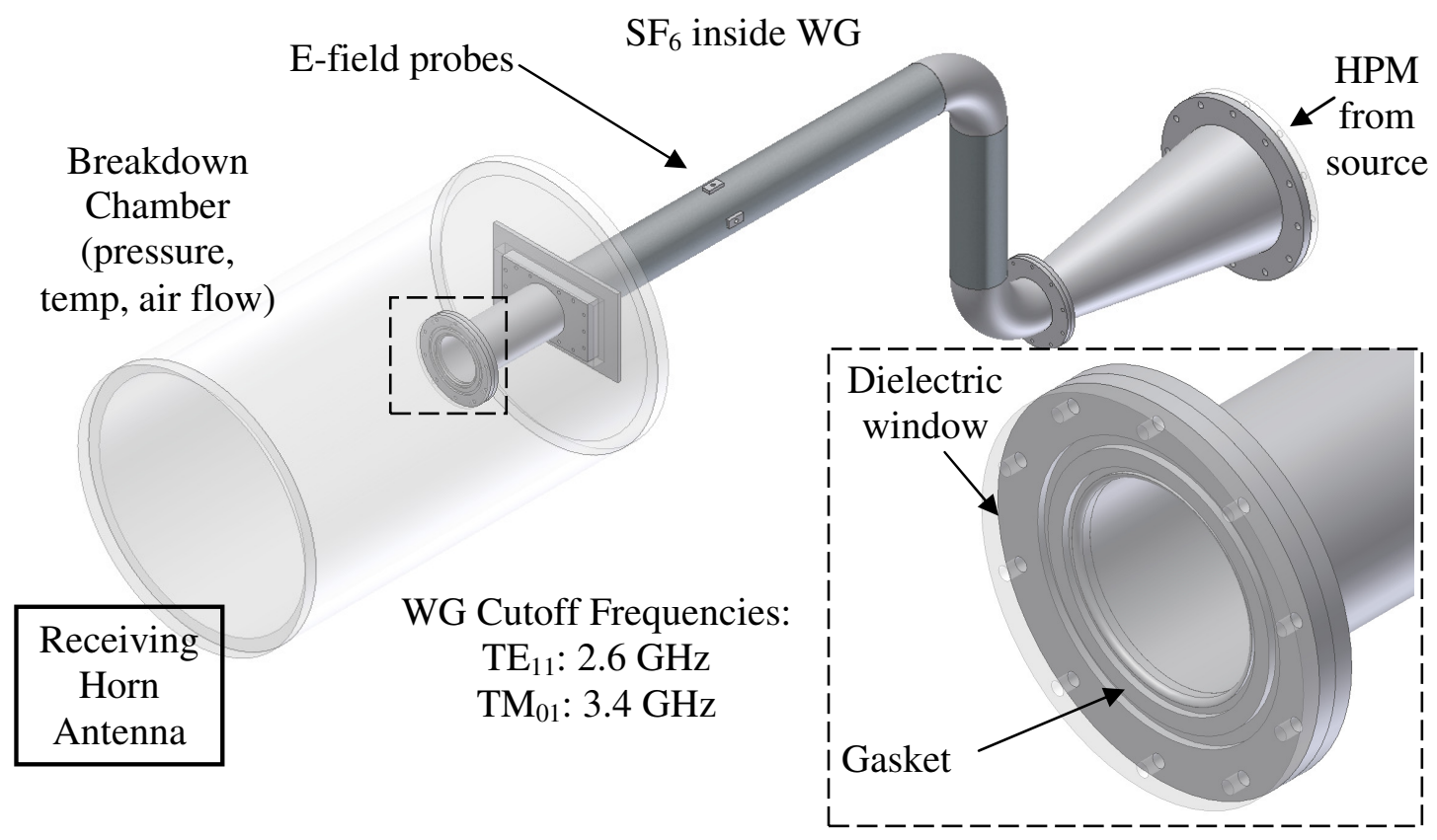

Figure 3.11: Conical transition, circular WG, exit flange, and breakdown chamber

\subsubsection{Conical Transition and Elbows}

In order to taper the 8" vircator chamber opening down to the required 2.6" inner diameter WG a conical transition was designed and constructed out of $1 / 4$ " aluminum sheeting that was rolled and welded to custom machined flanges. Several lengths were simulated in HFSS to minimize the $S_{11}$ reflected scattering parameter, which was found to be less than $-20 \mathrm{~dB}$. A section of $\mathrm{WG}$ with two $90^{\circ}$ elbows was fabricated due to dimensional constraints, but was also found to have a $S_{11}$ scattering parameter of less than $-20 \mathrm{~dB}$. The effects of these irregular WG sections on the field distribution is somewhat unclear, but the mode is still the dominant $\mathrm{TE}_{11}$ since that is the only mode that can propagate in the $\mathrm{WG}$ at $3 \mathrm{GHz}$.

\subsubsection{Circular WG}

A 2.5' section of WG made from commercially available aluminum pipe connects to the elbow section and terminates at the dielectric window where flashover occurs. The sections of the WG were welded to custom machined flanges and care was taken to design and weld the flanges such that welding seams on the inner surfaces were minimized. Some inner seams were required, especially on the conical transition, 
in which case they were sanded down to be as smooth as possible. Connections for a pressure gauge and Poly-Flo tubing are located on the straight section of the WG so that the WG can be filled with $\mathrm{SF}_{6}$ to prevent flashover on the inside of the WG. Efield probes are also located on the WG to measure the fields inside the WG and will be discussed later in the chapter.

The dielectric window is bolted to the end of the last WG section using nylon bolts to avoid triple points across the flashover surface. A field shaping gasket is also used on the inside of the WG to minimize field enhancement caused by the triple point so that breakdown on the inside of the WG is avoided. The design was loosely based on successful designs from [25] and found to sufficiently inhibit flashover on the $\mathrm{SF}_{6}$ side of the WG so no further design optimization was required. Lexan was used as the dielectric window material, though previous research indicates that other polycarbonates yield similar results [28].

\subsubsection{Breakdown Chamber}

The cylindrical WG and dielectric window project out into the breakdown chamber such that the flashover surface is not influenced by electron injecting metallic structures. The breakdown chamber is constructed of clear acrylic and designed to control atmospheric variables such as gas pressure, composition, temperature, and air speed. The chamber pressure is controlled with a roughing pump and valves to bleed in air. Due to limitations of the roughing pump and leaks in the chamber the pressure reaches a minimum of 5 Torr, setting the lower limit for pressure dependence testing. The pressure is monitored by a Varian ConvecTorr Thermocouple Vacuum Gauge, but because of leaks in the system maintaining a constant pressure through the manipulation of pump and intake valves is sometimes difficult, introducing an inherent error of at least a Torr to pressure measurements. The chamber also has valves to bleed in gas species other than atmospheric air and connections to allow air to be circulated through the chamber by a fan and cooled with an ethanol chiller. Because the chamber is clear and flashover usually occurs too quickly to develop more luminous visible arcs, obtaining imaging of flashover events and measuring the 
luminosity with photomultiplier tubes is complicated by the presence of ambient light. A picture of the circator, circular WG, and breakdown chamber is shown in Fig. 3.12.

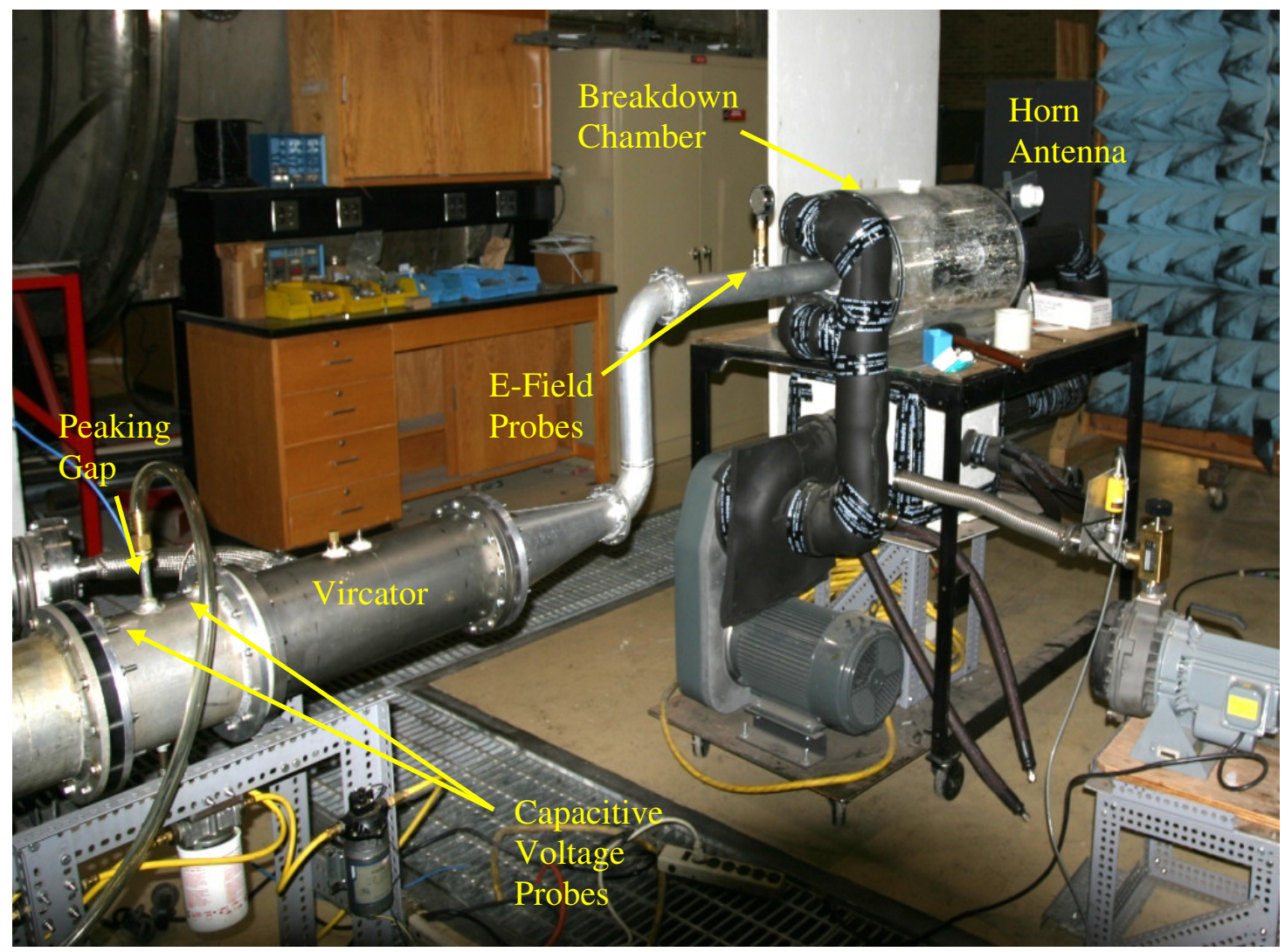

Figure 3.12: Vircator, circular WG, and breakdown chamber

\subsection{Diagnostics}

Diagnostics were designed with the intent of measuring the transmission line voltage before and after the peaking gap and the current through the vircator in order to monitor vircator operation. The HPM inside the WG and that transmitted past the window are also measured and compared to determine frequency, power, and pulse length of the HPM, as well as the delay time associated with the flashover event.

\subsubsection{PFL and Vircator Diagnostics}

Two capacitive voltage probes are located on either side of the peaking gap to monitor the charging of the PFL and its discharge across the peaking gap to the vircator. The probes were constructed by placing a layer of insulating Kepton tape and 
then a layer of copper tape in a horseshoe pattern around the inside of the peaking gap chamber such that a capacitance is created between the copper tape and the gap chamber and between the tape and the inner conductor. The tape is soldered to Type- $\mathrm{N}$ feedthroughs and covered with epoxy to hinder breakdown to the solder. A $500 \Omega$ resistor is connected externally and then a $50 \Omega$ coaxial cable connects the resistor to an Agilent Infiniium 12GHz, $40 \mathrm{GSa}$ /s digital oscilloscope. Such a high sampling rate is not required to accurately measure the output of the capacitive voltage probes, but it is the same oscilloscope used to directly sample other diagnostics measuring signals at the $3 \mathrm{GHz}$. A picture of the capacitive voltage probes inside the peaking gap is shown in Fig. 3.13.

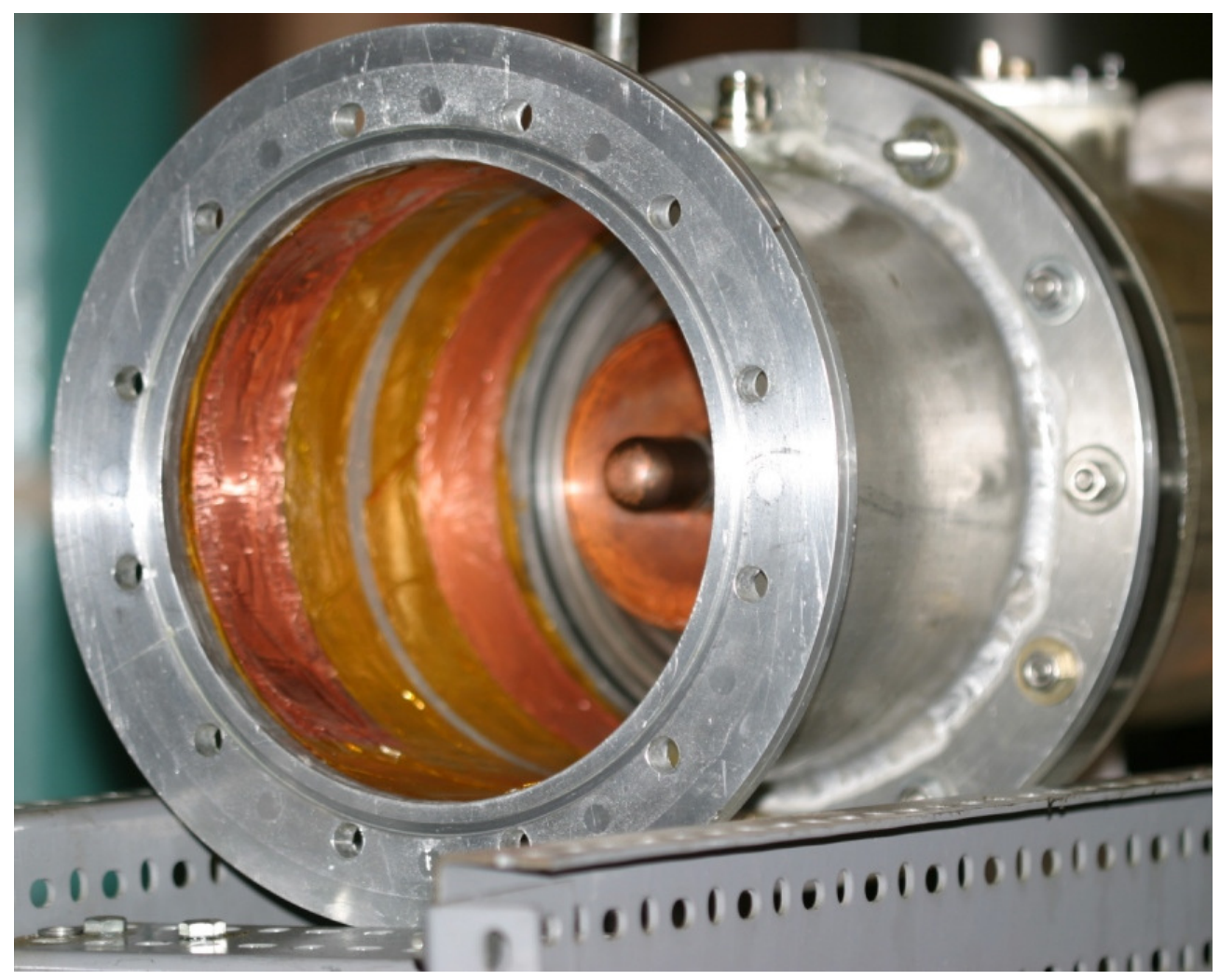

Figure 3.13: Capacitive voltage probes

The original peaking gap had capacitive voltage probes that were calibrated to measure the voltage on the line, but the peaking gap began to leak oil due to faulty welding seams and had to be remade. An initial attempt at constructing capacitive voltage probes on the new peaking gap failed after around a dozen shots due to air 
bubbles trapped in the spaces between the Type- $\mathrm{N}$ feedthroughs and the tape, which eventually caused the tape to break due to repeated exposure to pressure waves caused by breakdown in the peaking gap. The next attempt at fabricating capacitive voltage probes incorporated epoxy filling in the air gap between tape and feed throughs, which seems to have eliminated the problem. The probes were not calibrated and a divide ratio not determined, though the behavior of the peaking gap and time of breakdown can still be monitored, as shown in Fig. 3.14.

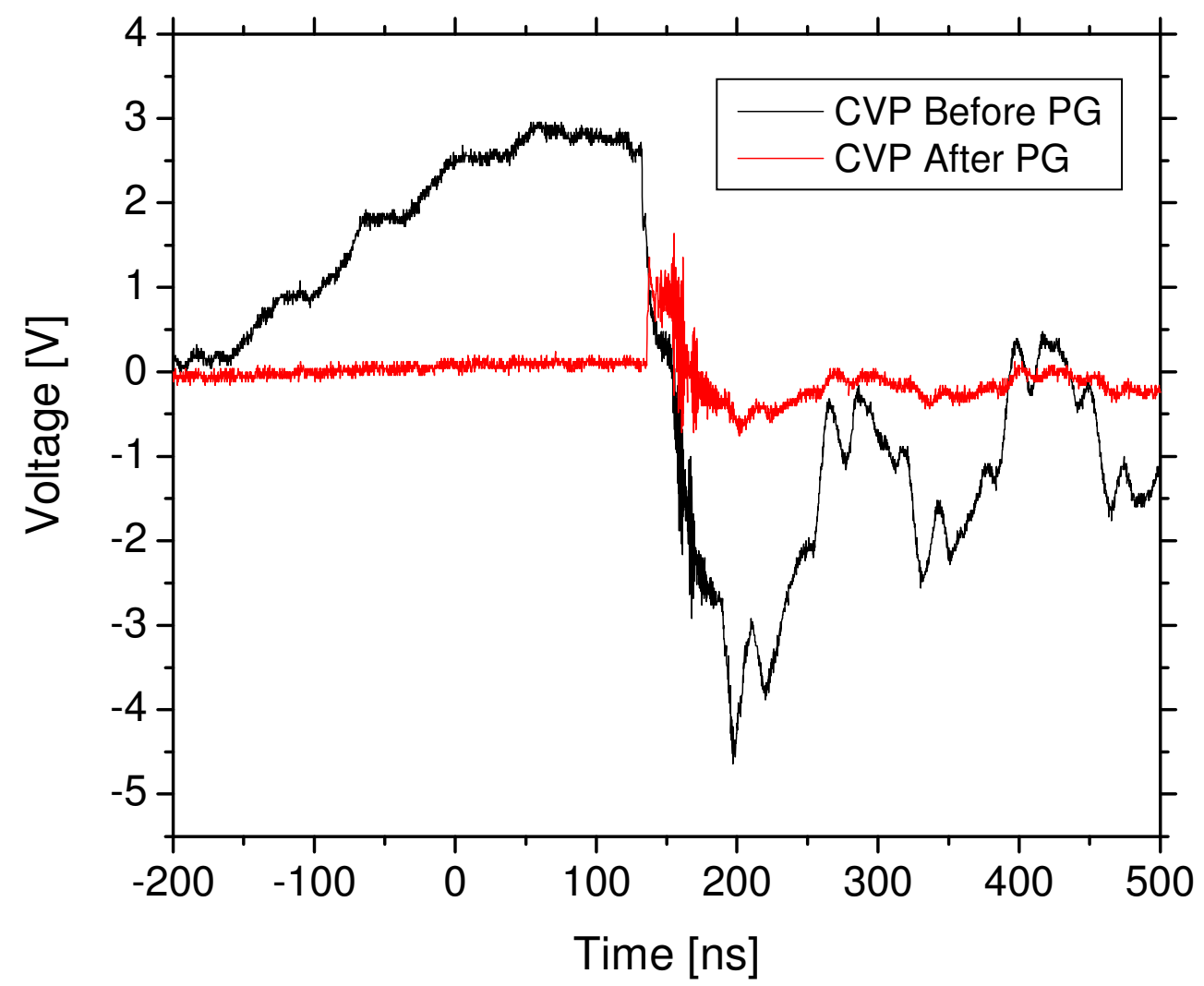

Figure 3.14: Capacitive voltage probe waveforms

The probe before the peaking gap can be seen rising over $350 \mathrm{~ns}$, at which point the peaking gap breaks down and the waveform abruptly falls to zero before overshooting and oscillating. At this time the probe on the vircator side of the peaking gap rises with a much shorter risetime. The waveform then returns to zero after $\sim 50$ ns, corresponding to the pulse length of the HPM. If the gap is too large, the waveform of the first probe has similar behavior for the first $350 \mathrm{~ns}$, but instead of quickly 
dropping to zero it slowly goes negative and then slowly rises to positive values again, at which point the peaking gap may or may not breakdown. This behavior is to be avoided as it limits the voltage applied to the vircator so these waveforms are closely monitored to ensure proper peaking gap spacing and oil cleanliness.

The current through the vircator is measured by a Rogowski coil around the cathode bolt, which produces a voltage proportional to the change in current. A typical measured waveform is shown in Fig. 3.15 along with its integral, corresponding to the actual current through the cathode.

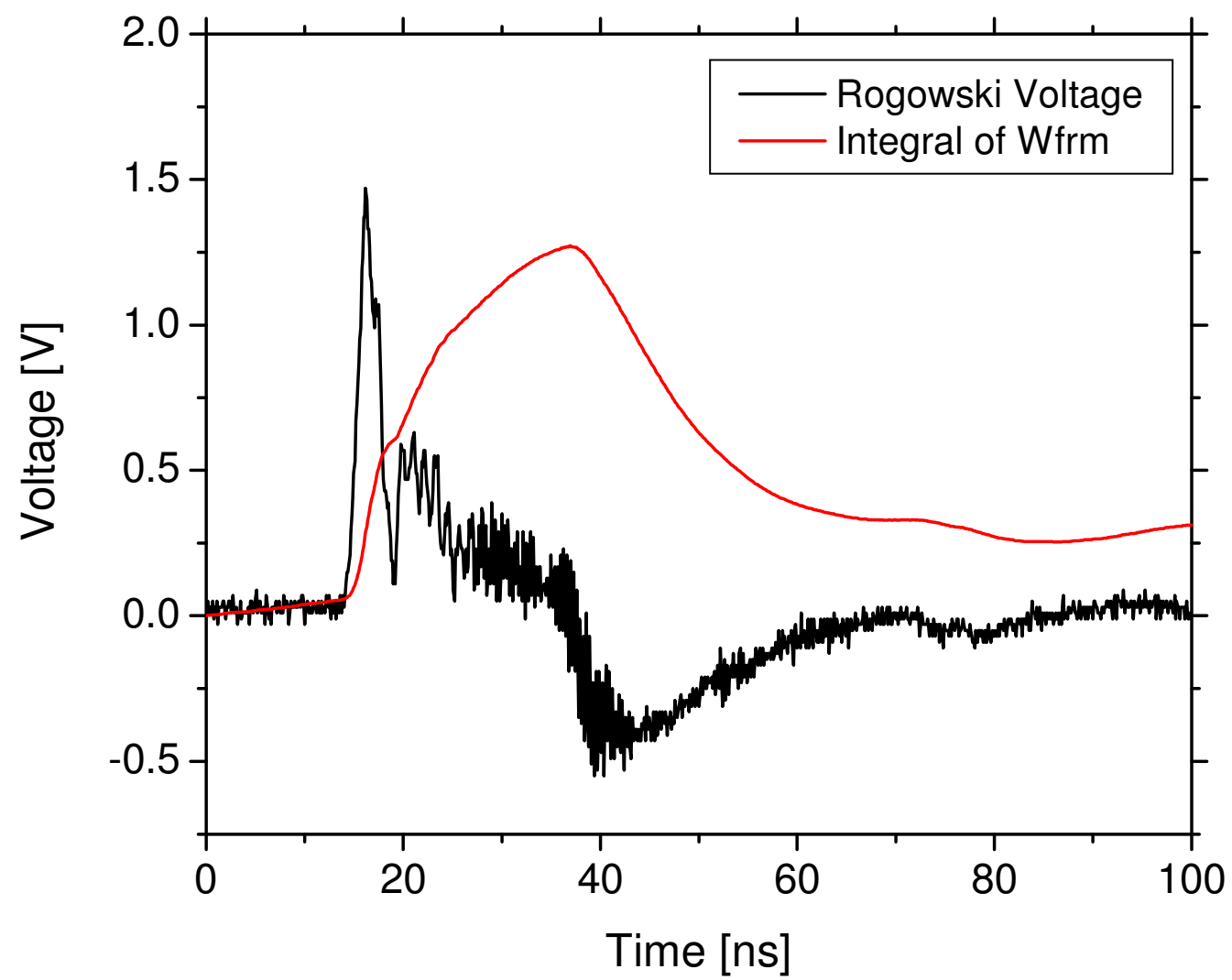

Figure 3.15: Rogowski coil waveform with integral

\subsubsection{HPM Diagnostics}

E-field probes are located on the straight section of the WG at a $90^{\circ}$ angle relative to each other, allowing the measurement of the HPM inside the WG. These probes were constructed by welding a curved pad to the top of the WG where holes 
were drilled and threaded to attach SMA connectors such that the center pin is flush with the inside of the WG. The voltage produced by these probes is attenuated and recorded by a $40 \mathrm{GS} / \mathrm{s}$ oscilloscope that directly samples the $3 \mathrm{GHz}$ signal. This voltage waveform is later processed and converted to power and then E-field levels, which will be discussed later in the chapter. The field configuration of the dominant $\mathrm{TE}_{11}$ mode is shown in Fig. 3.16 along with a picture of the SMA connectors. The field distribution is such that the E-field is mostly vertical, but bends out on the sides such that it is perpendicular to the WG surface. One would expect the top probe to measure the incident HPM, while the side probe would only have noise, though in reality some voltage is still measured on the side probe. This phenomenon will be further discussed in a later chapter.

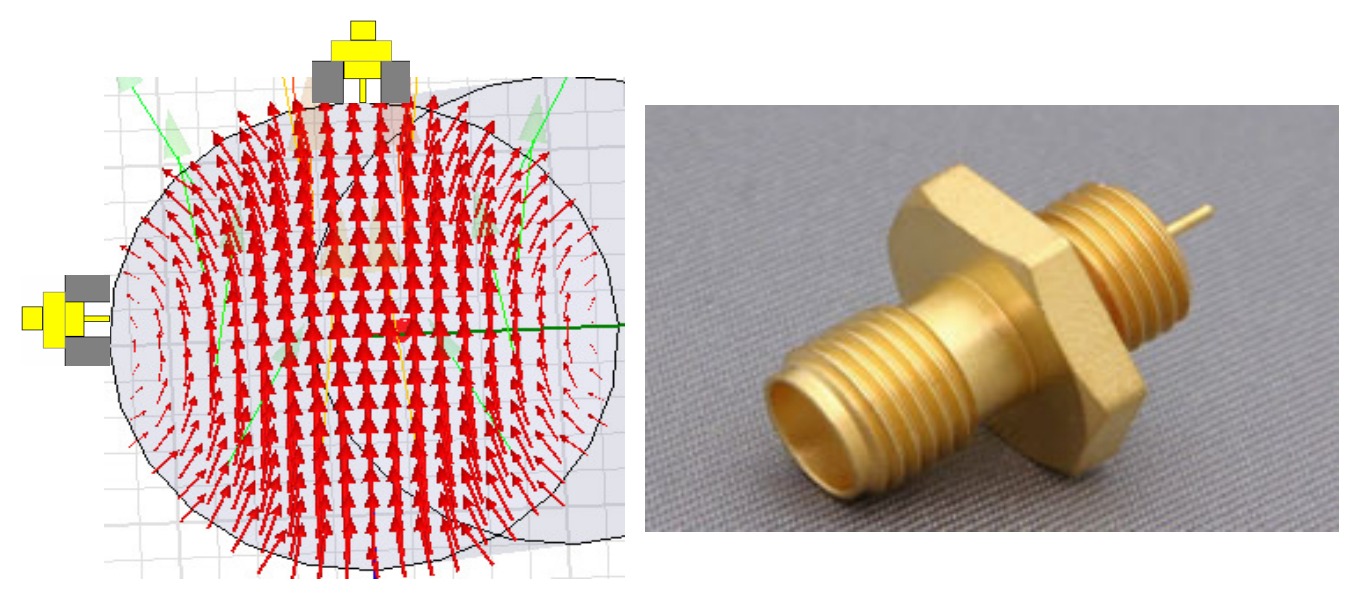

Figure 3.16: SMA E-field probes with WG field distribution

A receiving horn antenna from is located outside the breakdown chamber, about 2' from the window, so that the HPM transmitted past the window can be measured. The antenna is from Advanced Technical Matericals and has a nominal gain of $15 \mathrm{~dB}$ and is oriented vertically such that it is parallel to the $\mathrm{A} / \mathrm{K}$ gap of the vircator and the field distribution in the WG. In the absence of breakdown, the antenna waveform is in fairly close agreement with the top E-field probe, but with some differences due to the behavior of the HPM inside the WG.

When surface flashover occurs, plasma forms on the dielectric surface, causing the incident HPM to be absorbed and/or reflected. This is observed on the antenna 
waveform as a sudden drop in incident HPM. This drop in voltage is easily observable at low pressures when breakdown develops quickly and fully suppresses HPM transmission, but it is more difficult to determine breakdown time at higher pressures when breakdown takes longer to develop and only attenuates the transmitted signal. This is even more complicated by the presence of peaks and valleys in the envelope of the HPM, which can easily be mistaken with the occurrence of flashover. The method of calibrating the E-field probes will be discusses in the next subsection.

\subsection{Calibration}

Calibration of the capacitive voltage probes and cathode Rogowski coil were not undertaken as they were primarily used to monitor vircator operation by observing their timing and relative magnitudes. E-field probes located on the WG were calibrated in order to measure power inside the WG from the voltage recorded by Efield probes. The relationship between WG power and the effective E-field across the dielectric was also determined so delay times and other variables can be compared with the E-field in the region where breakdown is initiated.

\subsubsection{E-Field Probe Calibration}

The relationship between E-probe voltage and WG power was found by exciting the WG with a known, low-power microwave and then measuring the voltages at the E-field probes. A Hewlett Packard 8719C network analyzer was used to generate a $3 \mathrm{GHz}$ signal, which was amplified by an Amplifier Research 10S1G4A $\mathrm{RF}$ amplifier. The amplifier was needed because the E-field probes are designed to interfere with HPM propagation as little as possible so they pick up only a small amount of the $5 \mathrm{dBm}(400 \mathrm{mV}$ on a $50 \Omega$ cable) generated by the network analyzer (-54 dB plus $-2.8 \mathrm{~dB}$ for cable attenuation). The amplifier power was determined by attenuating the signal and measuring the voltage, which was used to calculate power. The WG was excited by feeding the amplified signal into a coaxial to rectangular WG adapter, since coaxial to circular WG adapters are not commercially available. The WR-284 adapter was connected to the circular WG with a custom circular to rectangular WG transition. See Appendix B for a description of the design of the 
transition. The transition was modeled in Ansoft HFSS and found to transmit 99\% of power. The $S_{11}$ reflected parameter of the actual transition was measured with the network analyzer and found to be less than $-20 \mathrm{~dB}$. The flanges and WG sections were machined from aluminum with a smooth finish and welded together such that the seams are on the outside surfaces of the transition. The R/C transition and calibration setup are depicted in Figs. 3.17 and 3.18 respectively.

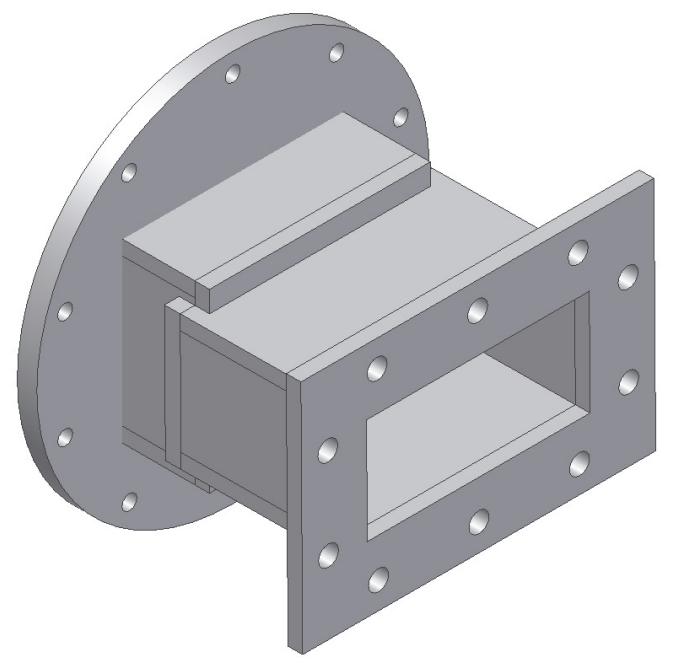

Figure 3.17: Rectangular to circular WG transition

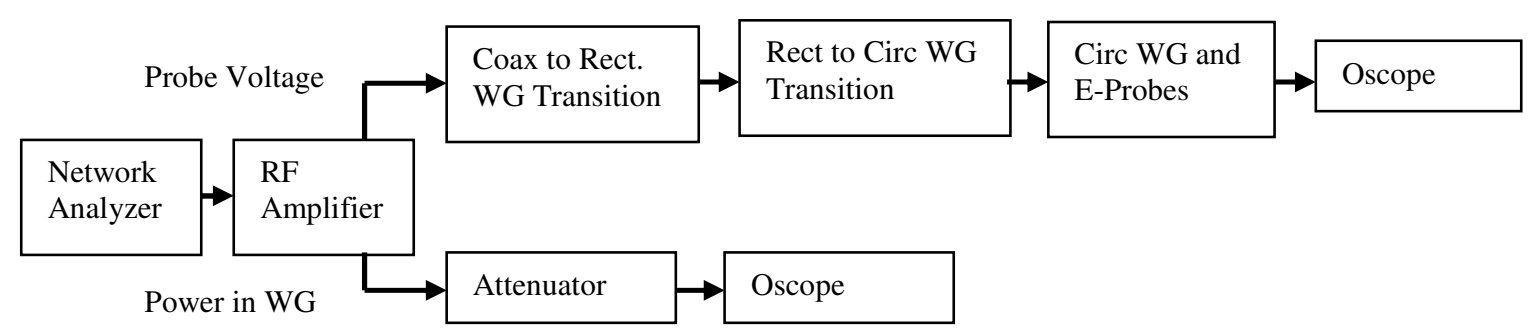

Figure 3.18: Preliminary calibration diagram

This calibration method was found to be inaccurate because the E-field probes are bidirectional and measure the sum of the incident and reflected E-fields at that point so the voltage measured is due to both the incident wave of a known power and the wave reflected by the window. The roundtrip delay time between the probes and window was calculating using the group velocity and the physical dimensions of the WG and found to be $\sim 7 \mathrm{~ns}$. This is much less than the risetime of the network 
analyzer, so a microwave switch (Mini-Circuits ZFSWA-2-46) with a 2 ns risetime was used to switch the signal so that the probe voltage can be measured before the reflected wave interferes. The fast rising signal is then amplified and used to excite the WG as shown in Fig 3.19.

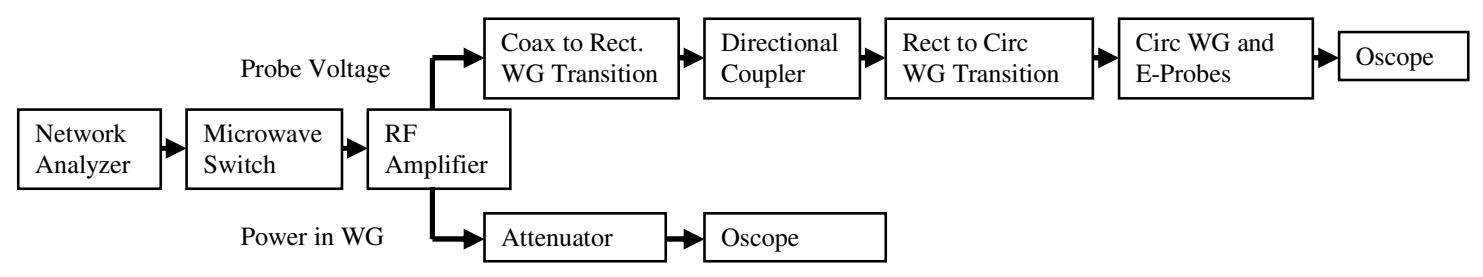

Figure 3.19: Final calibration diagram with switch

An E-probe waveform generated by the calibration setup with the switch is shown in Fig 3.20. An initial rise and then a drop in magnitude over the next several ns can be observed, which corresponds to the reflected wave beginning to cause a drop in the probe voltage, so only the voltage over the first $5 \mathrm{~ns}$ is used to determine the calibration. 


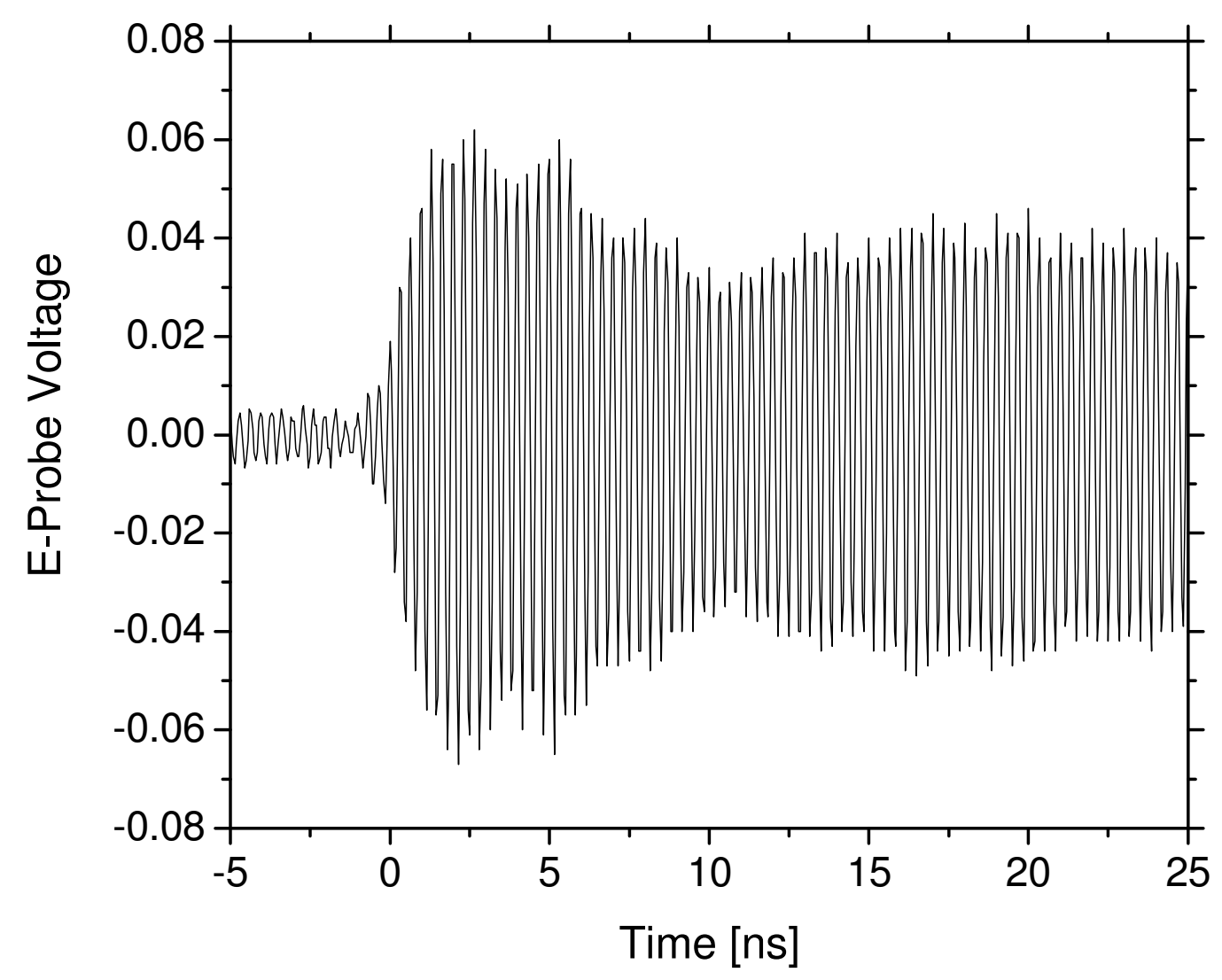

Figure 3.20: E-probe waveform for calibration with switch

A directional coupler was also placed between the coax to WG adapter and the rectangular to circular WG transition to ensure that the incident wave has a flat top and to check the reflection from the WG transition. A diagram of this setup is depicted in Fig. 3.21 and a picture in Fig. 3.22. 


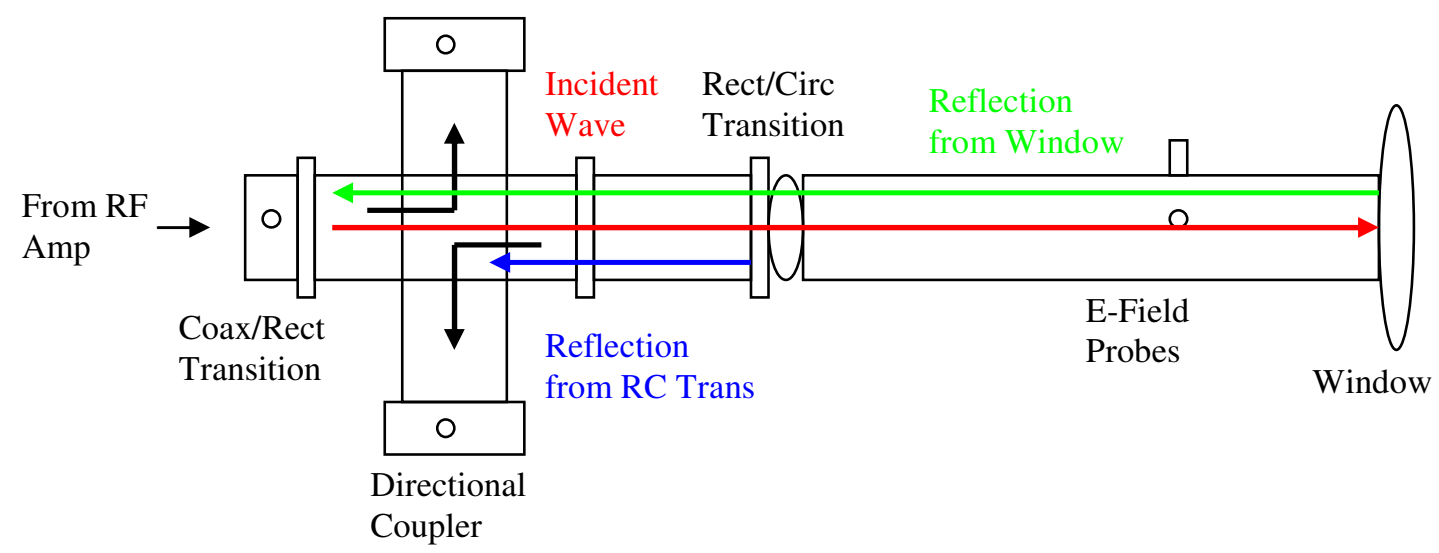

Figure 3.21: Calibration diagram with directional coupler

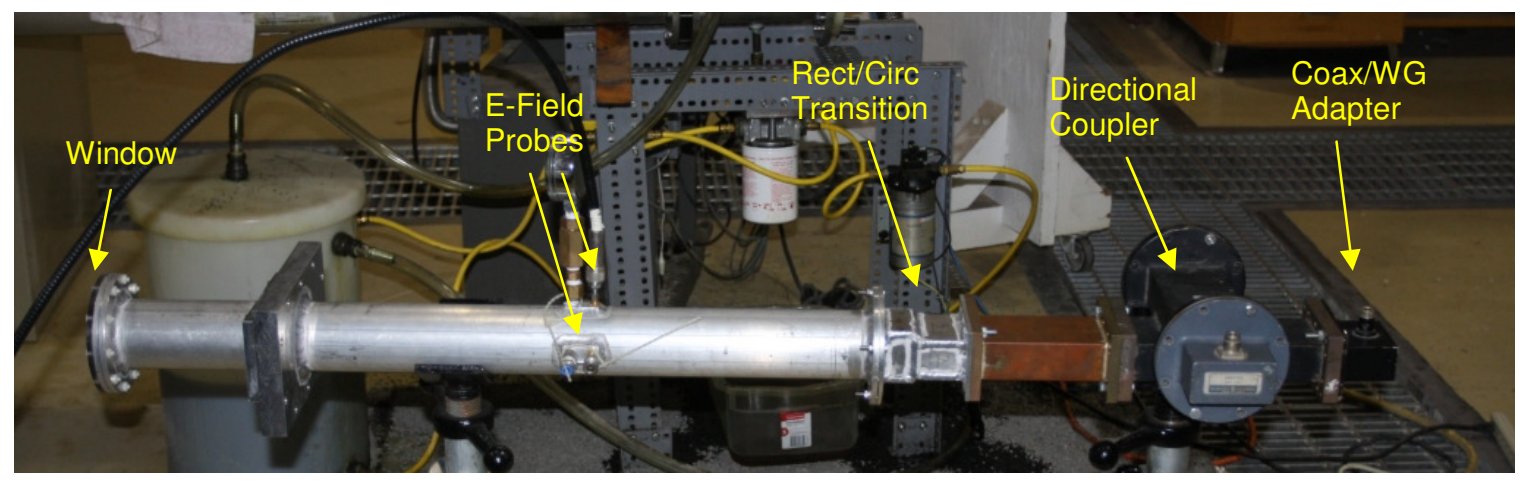

Figure 3.22: Actual calibration setup

The forward and reflected waveforms are shown in Fig 3.23. The forward waveform in verified to have a flat top as initially assumed. After a few ns the wave reflected by the rect/circ transition appears at the reflected port but it is barely above the noise threshold, as predicted by simulations and the scattering parameters measured with the network analyzer. After 25 ns the wave reflected by the window appears at the reflected port, which causes a sharp increase in the reflected waveform. 


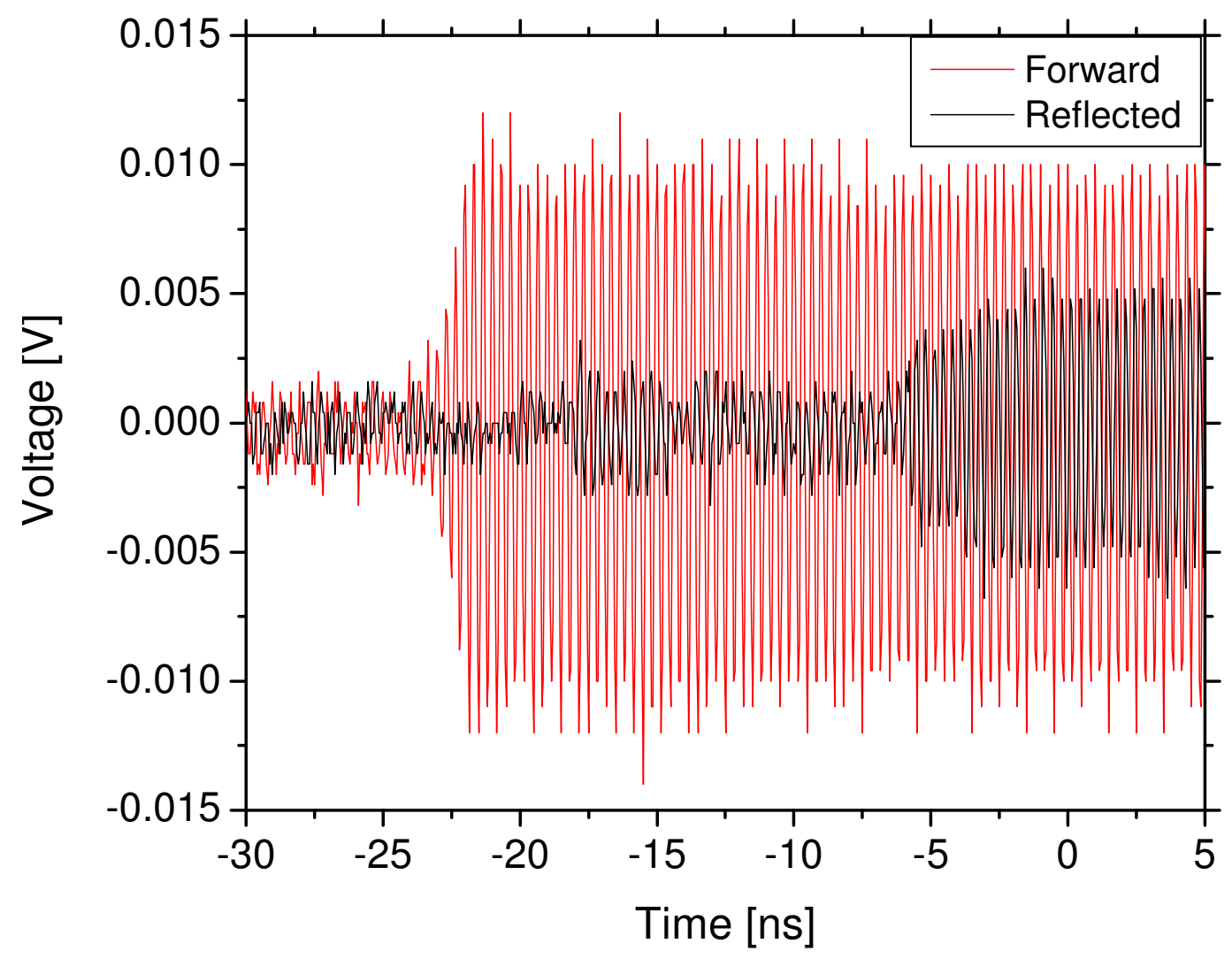

Figure 3.23: Directional coupler forward and reverse waveforms

The probe and amplifier waveforms were then recorded over a range of amplifications and used to calculate power levels and corresponding probe voltages. These values were then used to calculate a square relationship between WG power and probe voltage, which is plotted in Fig. 3.24. The equation for this relationship was calculated to be:

$$
P_{W G}[W]=1243 \cdot\left(V_{\text {probe }}[V]\right)^{2}
$$

where WG power is in watts and probe voltage is in volts. 

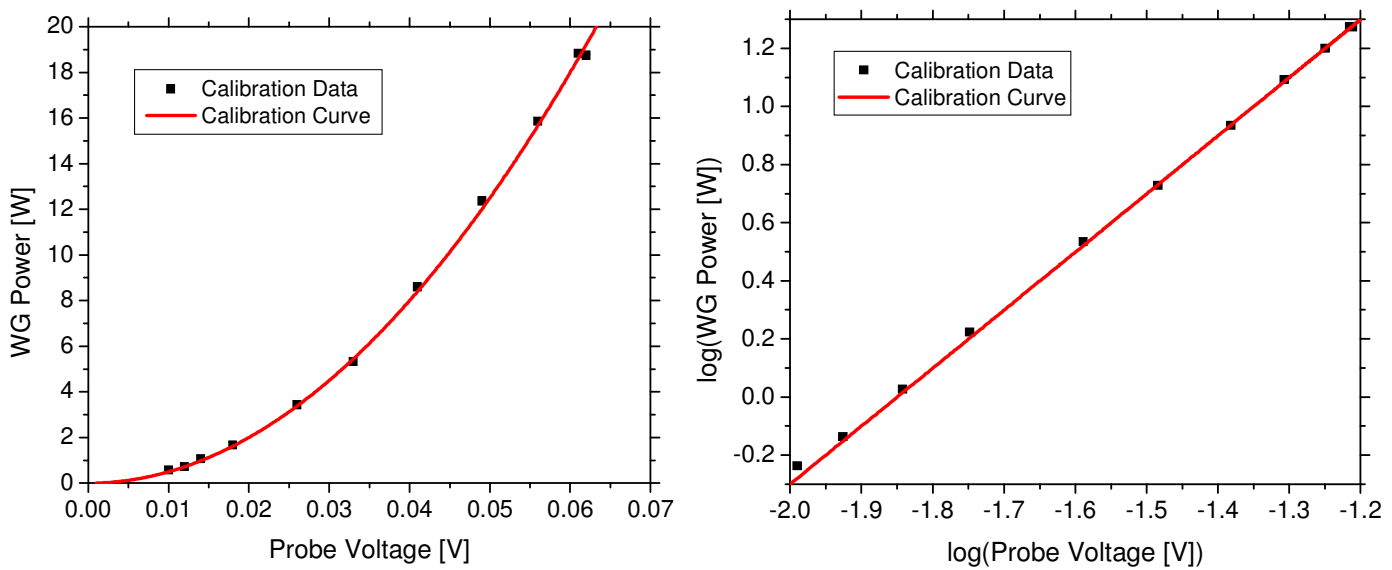

Figure 3.24: E-field probe calibration curves

\subsubsection{WG Power to Flashover E-Field}

Now that the calibration curve for the E-probes has been determined, a relationship between WG power and the E-field across the window is needed. Ansoft HFSS simulations were developed for the experimental geometry to determine this relationship. Because the HPM is radiated out at the window, a radiation boundary was used to envelop the WG, taking care that it was large enough to mimic open air radiation. The WG was then excited by a waveport on the side of the WG opposite the window. The effects of the conical transition and elbows were neglected as the probes were calibrated to measure the power at that point in the WG. In order to check the accuracy of this model, the E-fields inside the WG, away from the window, were plotted and found to be in close agreement to the field distribution and magnitude predicted by a $\mathrm{TE}_{11}$ mode excited by $1 \mathrm{~W}$ of power and shown in Fig. 3.25. 

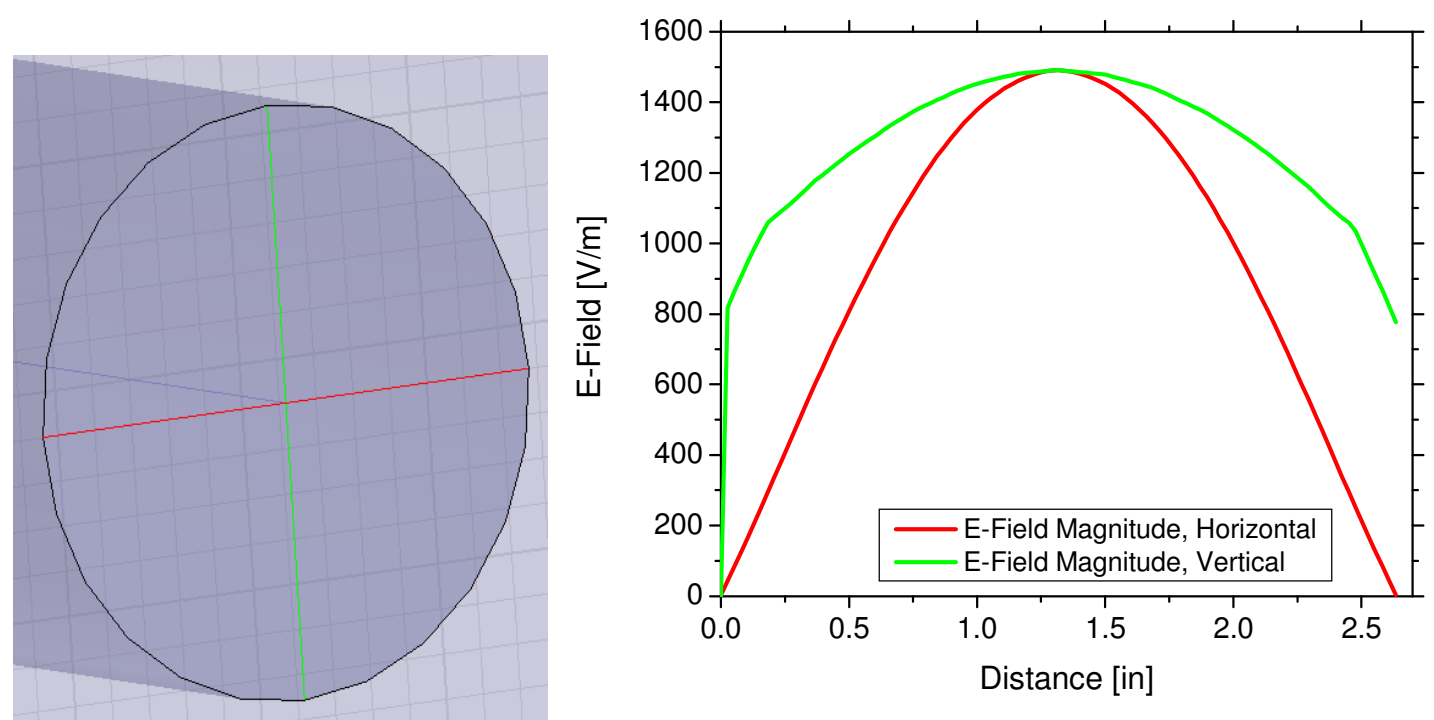

Figure 3.25: E-fields inside WG, away from window, excited by $1 \mathrm{~W}$

This behavior is expected considering the field distribution for a $\mathrm{TE}_{11}$ mode seen in Fig. 3.16. Both plots are highest at the center of the WG where they intersect and then decrease with radius but the red horizontal plot reaches zero at the edges of the WG whereas the green vertical plot only decreases to a value of over half the maximum E-field. There is a noticeable break in the smooth green plot around 0.25 " away from the edge of the WG, which is thought to be due to a reflection from the impedance mismatch at the window. The E-fields were also plotted inside the WG across the inner surface of the window and shown in Fig. 3.26. 

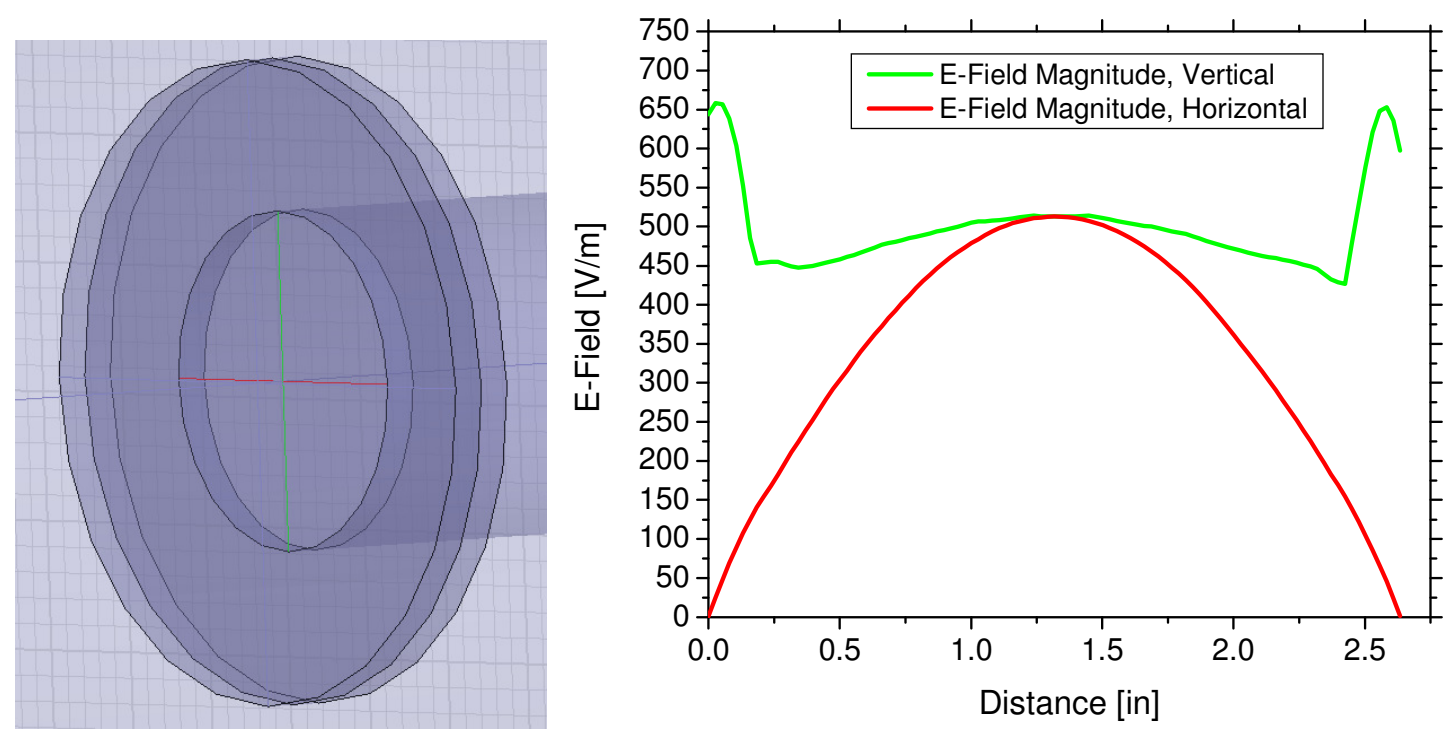

Figure 3.26: E-fields across inside of window, excited by $1 \mathrm{~W}$

These plots are similar to those further away from the window but the vertical plot increases along the edges of the WG instead of a smooth decrease from the maximum at the center. This behavior is also thought to be due to the reflection at the window, which is more pronounced at the edges of the WG. The plot of real interest is that of the E-fields across the outside of the window, where flashover occurs, and is shown in Fig. 3.27. 

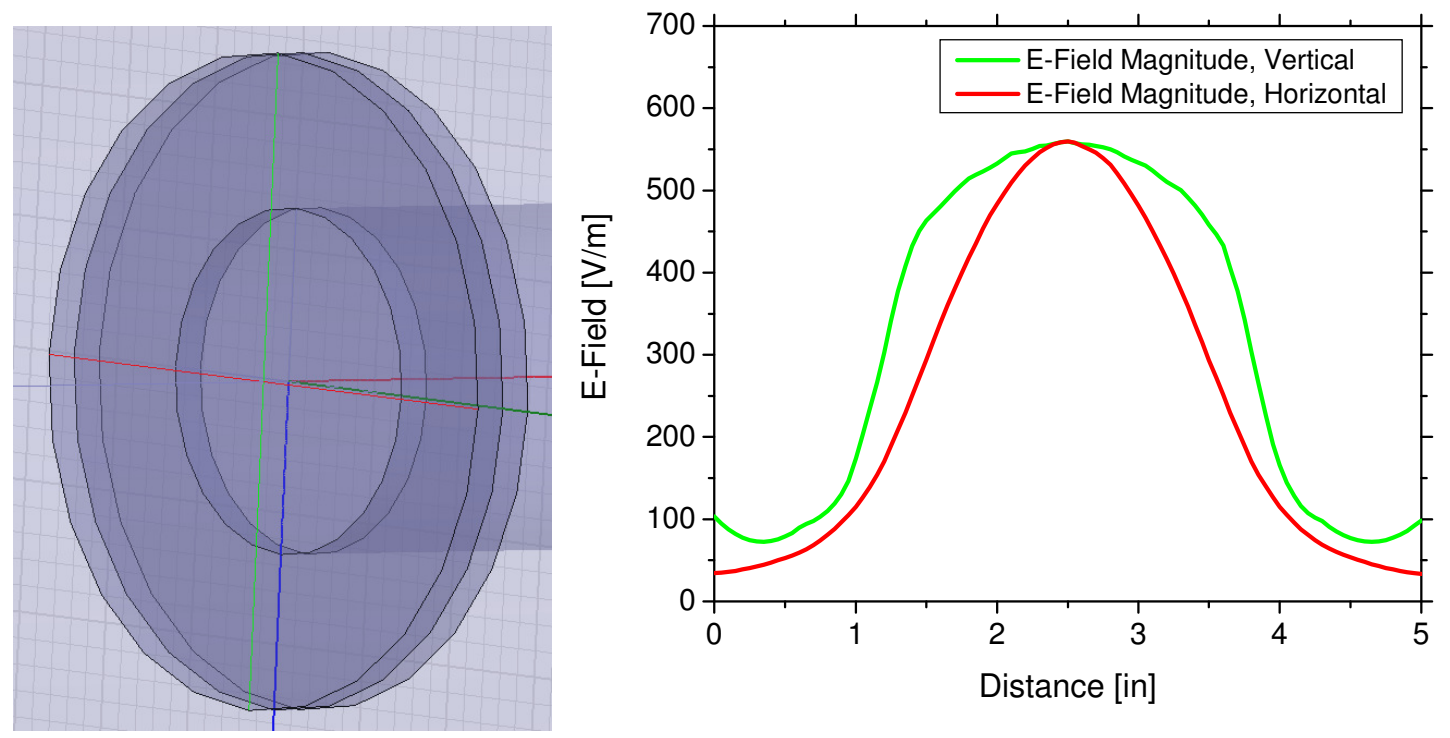

Figure 3.27: E-fields across outside of window, excited by $1 \mathrm{~W}$

This plot is of interest because it relates the power exciting the WG to the Efields across the surface. The field distribution is somewhat different than that inside the WG due to the reflection caused by the window itself and the impedance mismatch from WG to free space. The peak of the E-fields across the window is at the center of the WG and found to be $560 \mathrm{~V} / \mathrm{m}$, which corresponds to $1 \mathrm{~W}$ of WG power, resulting in a calibration equation of:

$$
E_{\text {window }}[V / \mathrm{cm}]=5.6 \cdot \sqrt{P_{W G}[W]}
$$

where the E-field across the window is in volts/cm and the power in the waveguide is in watts. This equation in conjunction Eq. 3.1 can then be used to calculate the E-field in the breakdown region across the window from the voltage measured by the E-field probes. A plot of the E-field at the center of the WG along the z-axis was also generated to check the validity of the model and is shown in Fig. 3.28. 


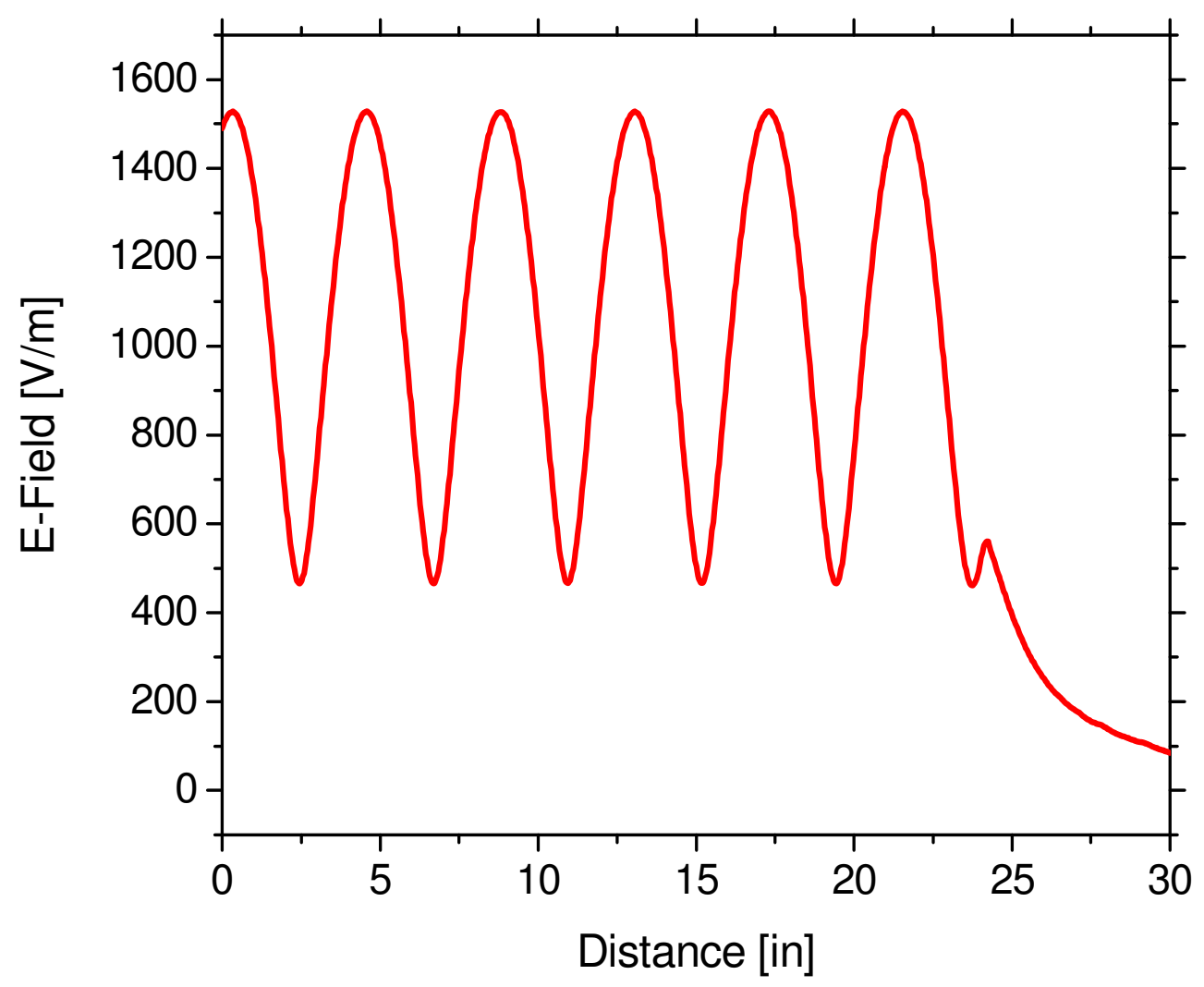

Figure 3.28: E-Field at WG center along z-axis, excited by $1 \mathrm{~W}$

This plot is also expected since the reflection at the window causes a standing wave pattern inside the WG. The window is located at $~ 25$ " where the small peak occurs. The bottom of the small peak is the inner surface of the window and the top of the small peak is the outer surface of the window. The E-field then decreases exponentially in free space.

Combining the relationship for E-probe voltage to WG power and that for WG power to E-field across the surface, a linear relationship between E-probe voltage and the peak E-field across the window was determined.

$$
E_{\text {window }}[V / \mathrm{cm}]=5.6 \cdot \sqrt{1243} \cdot V_{\text {probe }}[V]
$$




\subsubsection{Skewing in a Circular WG}

In the above simulations and calibration, it was assumed that the E-field is exactly vertical such that the top probe is the only one with an incident E-field. Since a circular WG has no dimensional constraints to keep the E-field vertical and due to the presence of transitions from the vircator to the straight WG section this assumption was investigated. The vircator was fired into the WG setup and the straight section of the WG was rotated such that the angles of the probes relative to the incident HPM varies. Four shots were taken at each angle and the absolute value of positive and negative peaks were plotted and shown in Fig. 3.29. Both positive and negative peaks were plotted because the E-probe waveform is not always completely symmetrical with zero. The top probe points reach a maximum around $10^{\circ}$ and then decrease to a minimum around $100^{\circ}$ and then increase again as the angle approaches $180^{\circ}$, while the side probe exhibits the opposite behavior. This indicates a skewing of $\sim 10^{\circ}$ and verifies that the $\mathrm{TE}_{11}$ mode is indeed propagating. Although a drop to zero would be expected when the probe is perpendicular to the center E-field, this does not occur in actuality due to coupling and possibly the presence of another $\mathrm{TE}_{11}$ mode with a different orientation, though the voltage ratio of parallel to perpendicular maximums is around 5:1, corresponding to a power ratio of 25:1. 


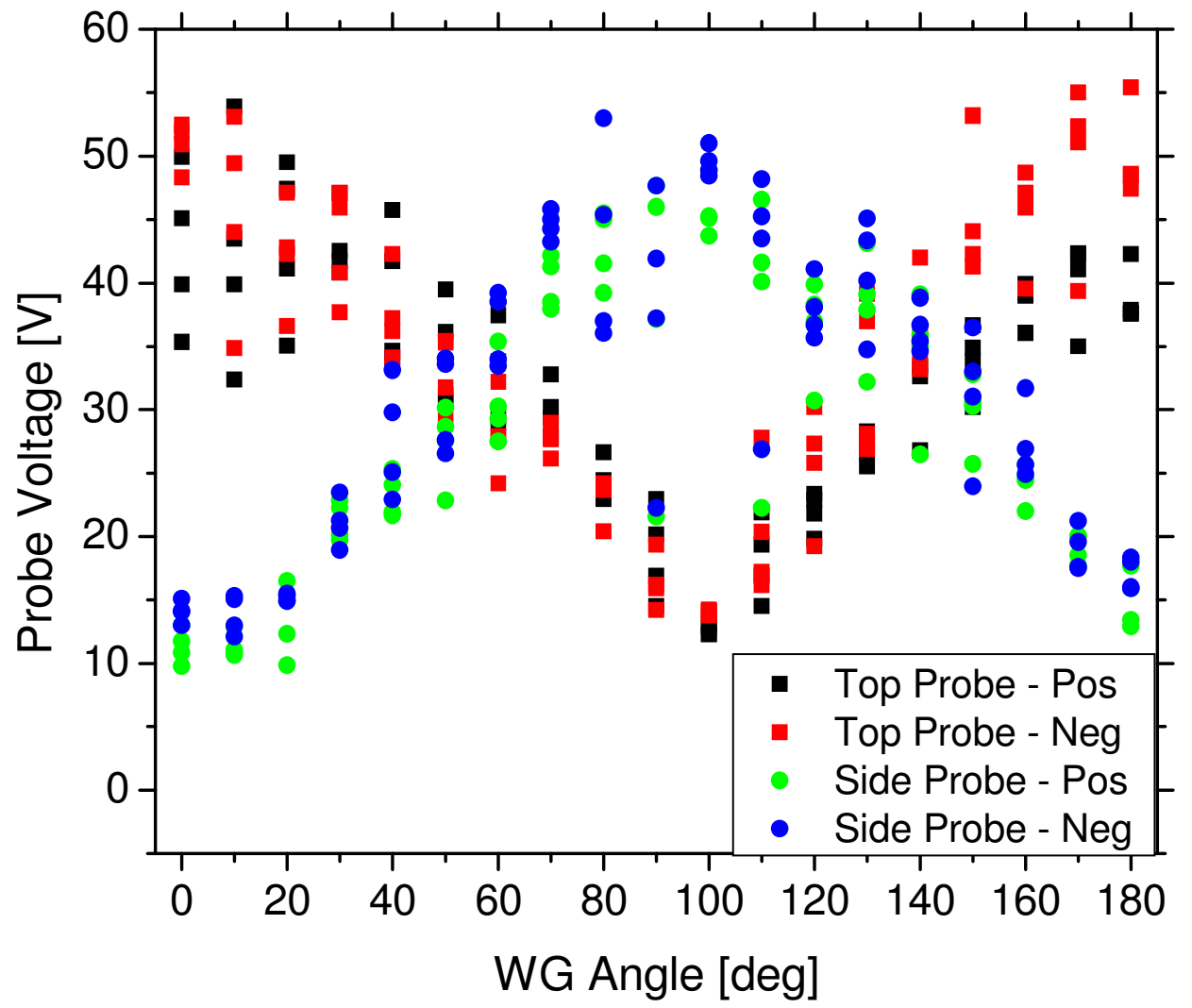

Figure 3.29: Top and side probe voltage magnitude variation with WG angular orientation

Because of this slight skewing, the voltage recorded by the top E-field probe is slightly less than the voltage that would be recorded if the E-field in the WG was completely vertical. To compensate for this effect, probe voltages are scaled by $1 / \cos$ $10^{\circ}$ (1.015) before processing, resulting in an overall calibration equation of:

$$
E_{\text {window }}[V / \mathrm{cm}]=200.396 \cdot V_{\text {probe }}[V]
$$




\section{Chapter IV}

\section{EXPERIMENTAL RESULTS AND DISCUSSION}

\subsection{Typical Waveforms}

Waveforms from the top E-field probe and the horn antenna in the absence of flashover (atmospheric pressure) are depicted below in Fig. 4.1. There is a definite correlation between the two waveforms, but the antenna measures only the HPM transmitted past the window, whereas the probe measures the incident HPM as well as that reflected from the window. This would not be as much of an issue if the pulse had a square shape with a flat top, but since the HPM has changes in magnitude over time, these reflections have differing effects on the measured waveform. At the beginning of the pulse, the probe waveform increases when the reflected wave reaches it, but at later times when the incident waveform decreases, the probe waveform does not due to the presence of the reflected wave. This behavior makes it somewhat difficult to calculate E-fields and delay times, so some processing and averaging is required to obtain accurate data. 


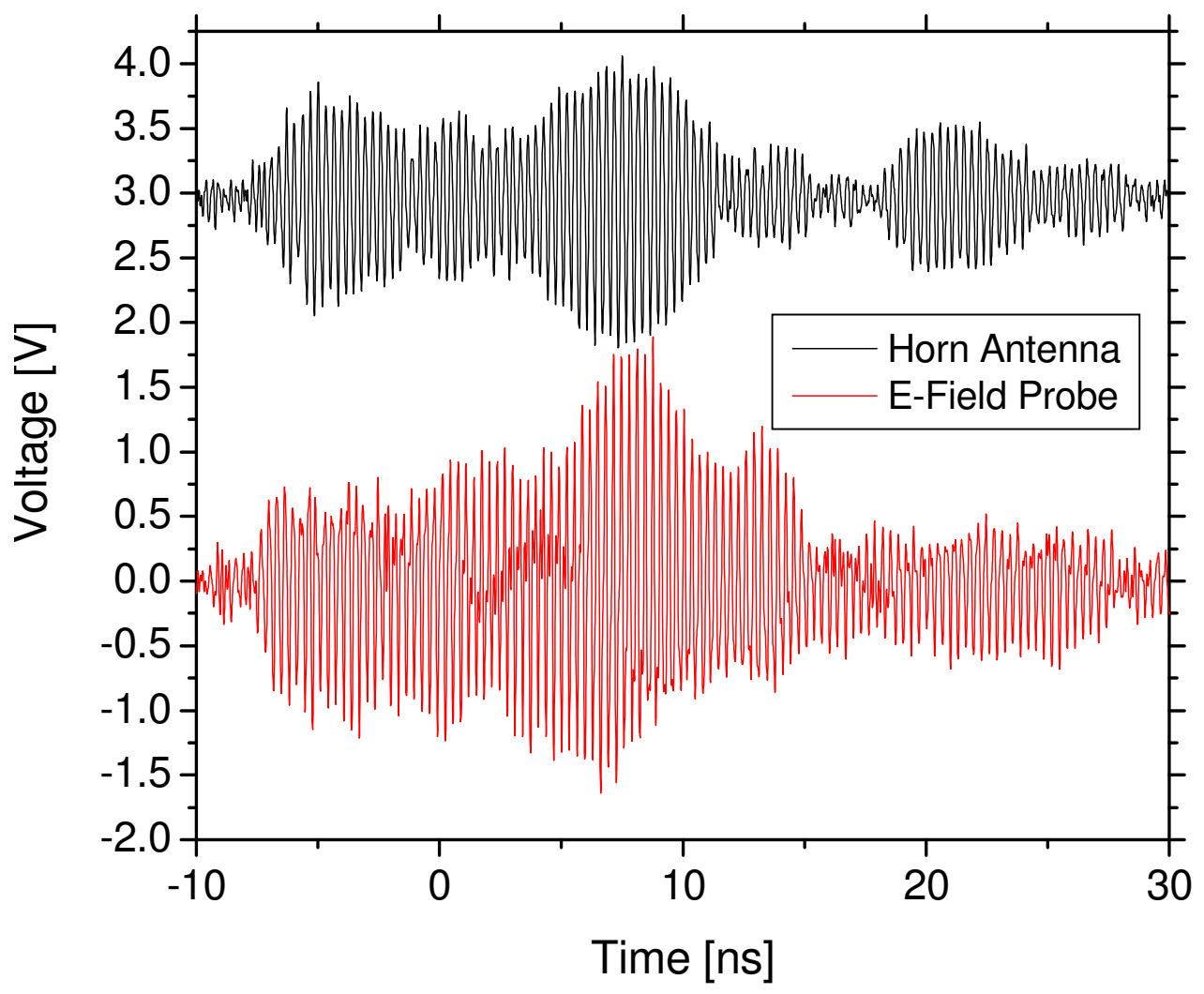

Figure 4.1: Typical probe and antenna waveforms, no flashover occurring

Waveforms of the probe and antenna differ markedly when flashover occurs. Because the developing plasma absorbs/reflects the incident HPM, the antenna waveform drops to zero once flashover occurs, while the probe waveform closely mimics the incident waveform. Fig. 4.2 depicts the probe and antenna waveforms in flashover at 5 Torr in air. The antenna waveform quickly goes to zero once flashover occurs at $-10 \mathrm{~ns}$. 


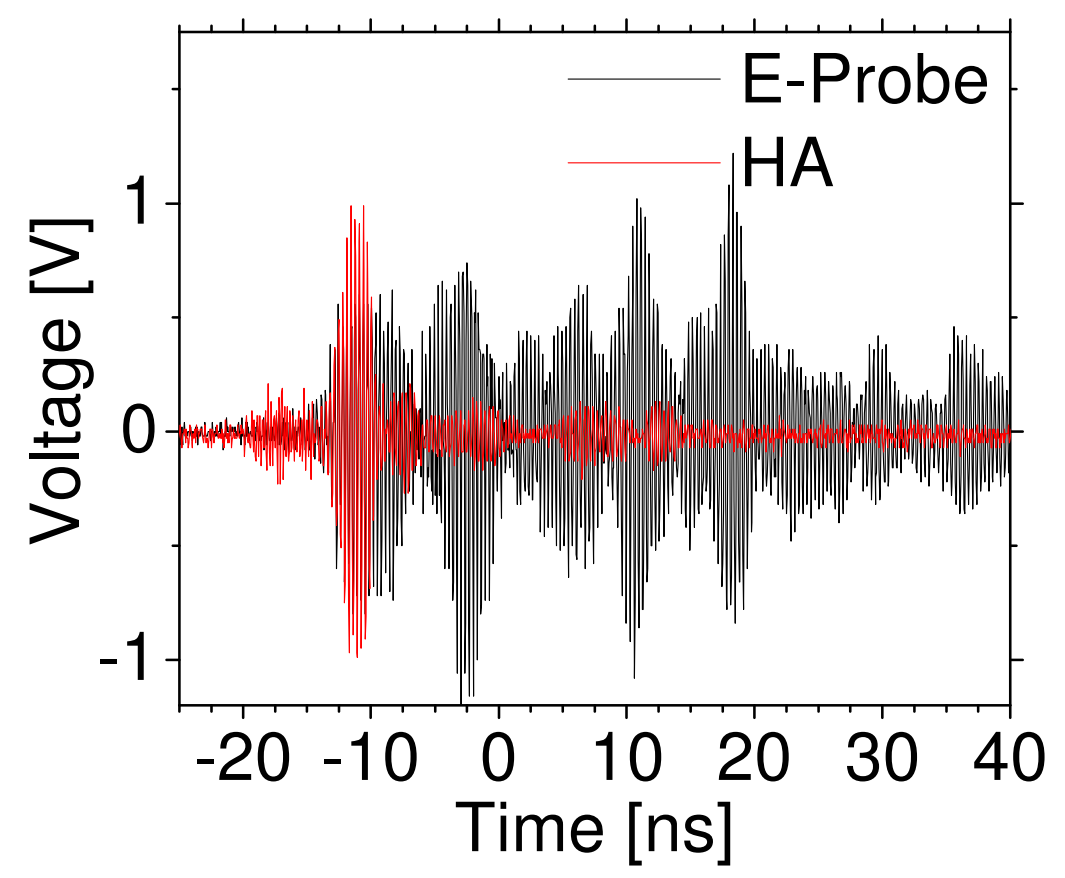

Figure 4.2: Typical probe and antenna waveforms, flashover at 5 Torr in air

This behavior allows for the determination of delay times by calculating the time between the rise of the waveforms and the absence of the antenna waveform. At low pressures, this determination is fairly straightforward, but at higher pressures flashover takes longer to develop and care must be taken not to mistake a drop in the incident waveform with flashover. This determination is also complicated at higher pressures because the HPM is not completely absorbed/reflected over a short period of time. Fig. 4.2 depicts probe and antenna waveforms over a range of pressures to indicate the relationship between delay time and pressure as well as the differing degrees of attenuation by the developing plasma. Also of note is the differing waveforms produced by the vircator, which vary significantly from shot to shot. 

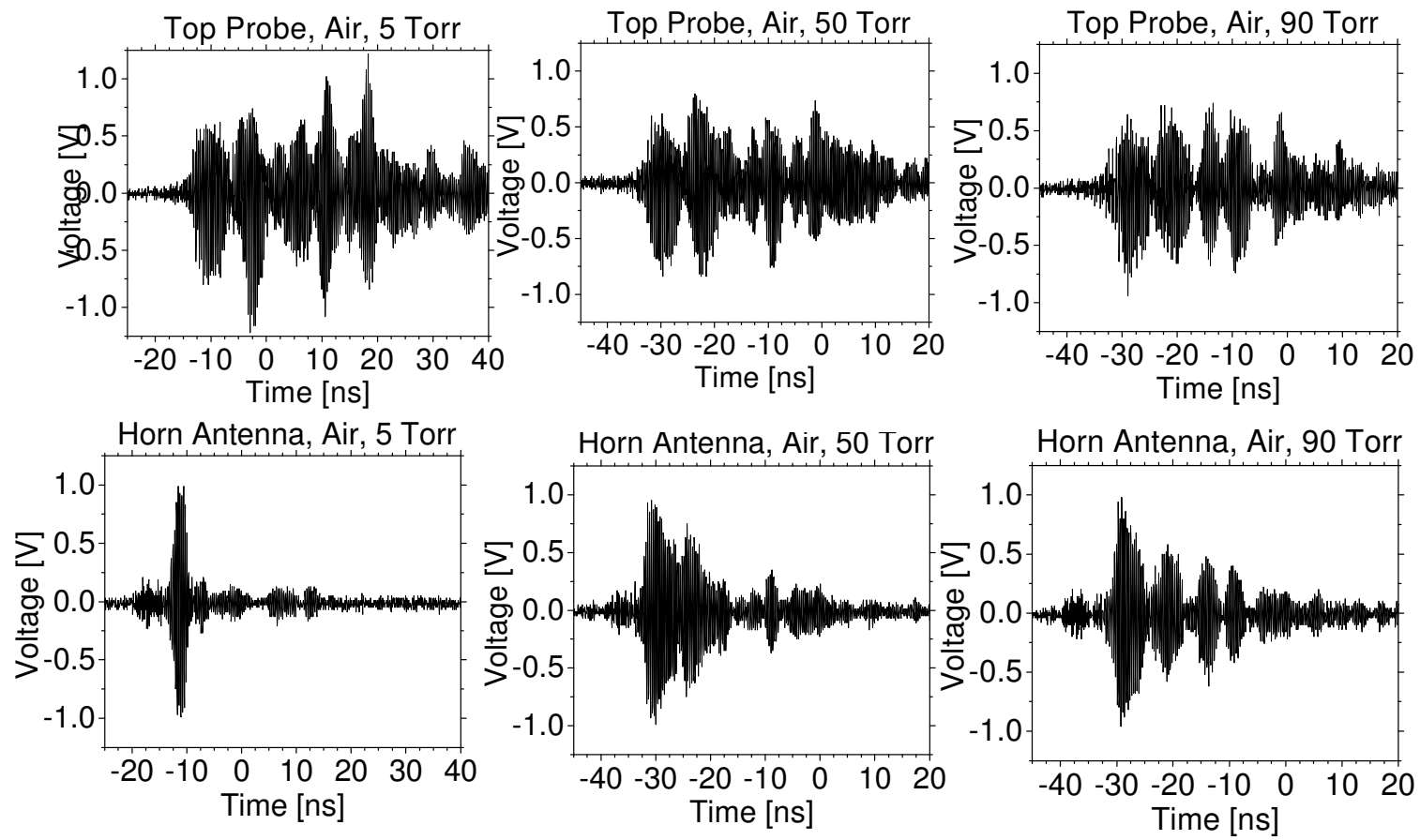

Figure 4.2: Typical probe and antenna waveforms of flashover at 5, 50, and 90 Torr

In order to determine the delay time associated with a flashover event, the probe and antenna waveforms must be compared to determine when the antenna waveform drops but the probe waveform does not. This is accomplished with a Mathcad program written to process this data. First the peaks of the waveforms are found, then curves are fit to those peaks to generate envelopes of the waveforms. These envelopes are then compared so that the time is found when the antenna envelope drops below the probe envelope. An alternate method of determining delay time is to find the time that the antenna signal drops below a certain threshold, but care must be taken to ensure that this drop does not correlate to a drop in the probe waveform as well. These algorithms were developed with the intent of processing a set of data all at once, but due to variations from shot to shot this has yet to be done. Processing data still requires someone to verify the delay times calculated by the program and occasional adjustments to the algorithms. 


\subsection{Delay Times}

In order to investigate the relationship between gas pressure and delay time, data was taken over a range of pressures from 5 to 90 Torr and delay times were calculated and averaged for each pressure. Both datasets exhibit a trend of increasing delay times with pressure, indicating that breakdown is taking place on the collision dominated right side of the Paschen curve. Since no pre-ionizing source is used, these delay times are the sum of the statistical and formative delay times. The statistical delay time is the time it takes for an initiatory electron to appear in the high field region, and exhibits some statistical variance from shot to shot. The formative delay time is the time it takes for an initiatory electron to multiply and produce breakdown. The large variance in these delay times cannot be solely attributed to variation in statistical delay times, but is also likely due to changes in the HPM waveform from shot to shot. This effect is more pronounced at higher pressures where delay times are longer and the shape of the pulse gains importance.

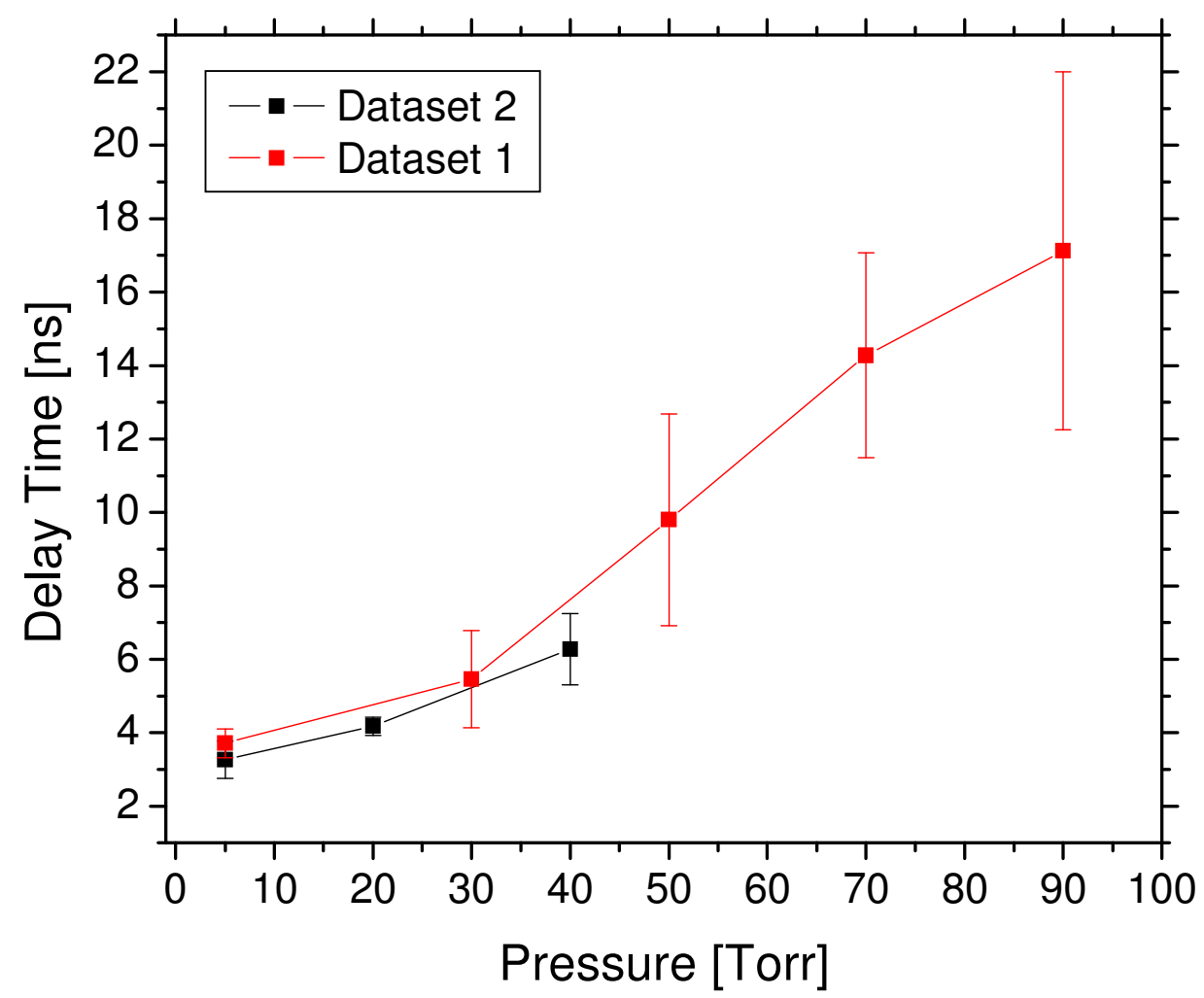

Figure 4.3: Delay times from 5 to 90 Torr 


\subsection{E/P vs $\mathrm{P} *$ tau Plots}

In order to compare this breakdown data to data taken with different experimental setups at different power levels and frequencies, the data is normalized by plotting the effective E-field divided by pressure against the delay time multiplied by pressure. This allows experiments at different power levels, frequencies, and pressures to be compared, but it does not take into account other differences in experimental setups such as the presence of a triple point or a pre-ionization source. The effective E-field is determined by first applying the calibration curve to the probe waveform and calculating the peak E-field. The effective E-field is then calculated from:

$$
E_{e f f}=\frac{E_{p e a k}}{\sqrt{2 \cdot\left(1+\frac{\omega^{2}}{v_{c}^{2}}\right)}}
$$

where $\omega$ is the angular frequency of the HPM and $v_{c}$ is the collision frequency of electrons with background gas molecules. For large ratios of $\omega / \mathrm{v}_{\mathrm{c}}$, electrons are accelerated in the opposite direction before collisions can occur and so are effectively oscillating back and forth, resulting in an effective E-field that is significantly smaller than the peak E-field. For small ratios of $\omega / v_{c}$, electrons experience many collisions before the field changes direction so the oscillating field has a lessened effect on the motion of electrons and the effective E-field becomes the rms value of the peak Efield.

In order to calculate the E-field at the time of breakdown, a calibration curve is applied to the E-probe voltage waveform to produce a waveform of E-field across the window over time. Since this field varies with the HPM waveform, an averaging method was used to average together the E-field values above $90 \%$ of the maximum. Other threshold values were also calculated, but the difference between values was not significant down to $75 \%$ of the maximum. This data is plotted in Fig. 4.4 along with data from the magnetron experiment, Yee (LLNL) [34], and Felsenthal and Proud [7]. 


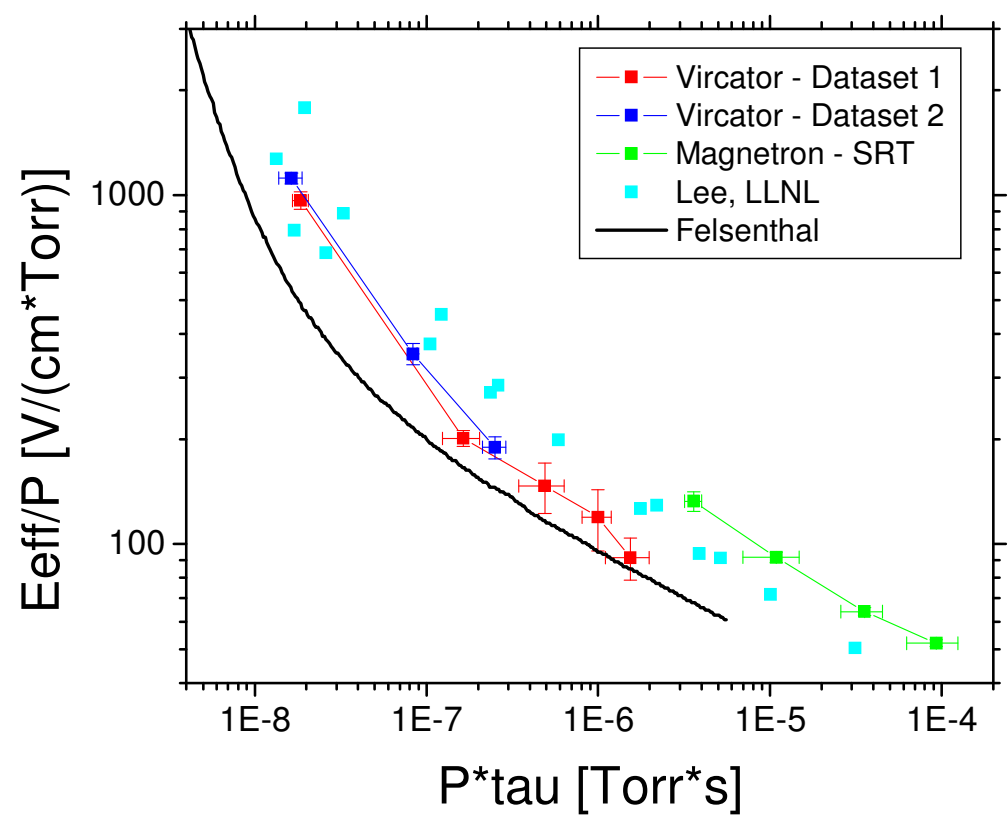

Figure 4.4: E/P vs P*tau

While all of these curves lie relatively close together, there are significant differences in experimental setups that account for differences in the data. The vircator and magnetron experiments have the most in common in that they both generate HPM surface flashover without a triple point or pre-ionization sources. The do differ in pulse length, power levels, and pressure ranges, resulting in the magnetron occupying a lower E/P range. Yee's experimental setup differs significantly from that for the vircator in that it is volume RF breakdown with a pre-ionization source. The Felsenthal curve likewise has significant differences in that it is unipolar breakdown with a pre-ionization source, but the Yee and Felsenthal data are in the same E/P range as the vircator data and the datasets do lie quite close together. It is difficult to make any further assertions about the significance of these differences due to the large variance involved in the current experimental setup.

\subsection{Imaging}

Imaging of the flashover event was also undertaken to get a better idea of how the process develops. Open shutter pictures were taken of breakdown in air and in 
argon and are shown in Fig. 4.5. The flashover images show that the flashover doesn't develop into visible arcs, but does produce a glowing plasma capable of reflecting/absorbing the incident HPM. This behavior is most likely due to the short pulse length and low pressure involved in this experiment.
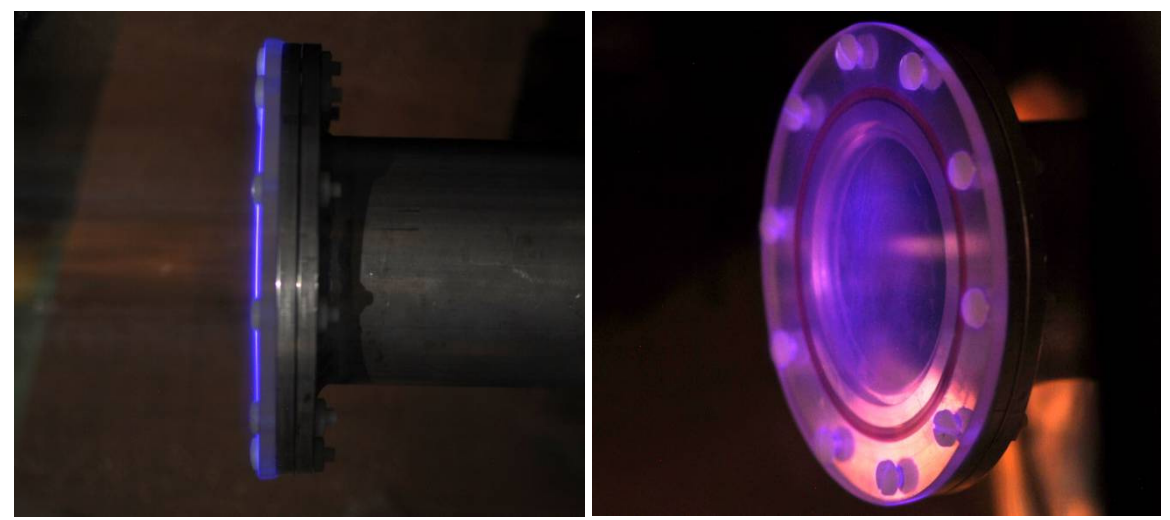

Figure 4.5: Imaging of Flashover in Air and Argon 


\section{Chapter V}

\section{Conclusions}

An experimental apparatus was designed and built to study HPM surface flashover without the influence of a triple point at high electric field amplitudes and short pulse lengths. Diagnostics were developed to measure delay times and E-fields aw wells imaging of the flashover event. Breakdown data was collected over a range with little to no available data found in literature. Although experimental setups found in literature differ somewhat from the current setup, breakdown data was found to closely correlate to previously published experimental data. Additional insight into the relative importance of mechanisms and processes involved is complicated by the inherent variance in the experimental setup, specifically the HPM generating vircator.

Future work with the current setup could shed additional light on the flashover process by investigating breakdown in other gases and by varying environmental parameters like temperature and humidity. A pre-ionizing source could also be incorporated to reduce the statistical delay time and allow for a better comparison with data in literature done under similar conditions. The primary suggestion for future work in this field is to use a more consistent HPM source. A vircator could be used again as it is relatively simple to design, but care must be taken to design the system to be more repeatable and less prone to variance. There is little doubt that understanding of this phenomenon is far from complete and hopefully this study will provide insight into this type of research and facilitate future work in this field. 


\section{REFERENCES}

[1] E. Nasser, Fundamentals of Gaseous Ionization and Plasma Electronics, Wiley Series in Plasma Physics, Wiley-Interscience, New York, 1971.

[2] Y. Raizer, Gas Discharge Physics, Springer-Verlag, New York, NY, 1991.

[3] J. M. Meek and J. D. Craggs, Electrical Breakdown of Gases, John Wiley and Sons, 1978.

[4] A. D. MacDonald, Microwave Breakdown in Gases, John Wiley \& Sons, New York, 1966.

[5] A. D. MacDonald, D. U. Gaskell, H. N. Gitterman, "Microwave Breakdown in Air, Oxygen, and Nitrogen," Physical Review, Vol. 130, No. 5, pp.1841-1850, June, 1963.

[6] L. Gould and L. Roberts, "Breakdown of Air at Microwave Frequencies," Journal of Applied Physics, Vol. 27, No. 10, pp.1162-1170, October 1956.

[7] P. Felsenthal and J. M. Proud, "Nanosecond-Pulse Breakdown in Gases," Physical Review, Vol. 139, No. 6A, pp.A1796-A1804, September 1965.

[8] D. Anderson, M. Lisak, and F. Wising, "Short High Power Microwave Pulse Propagation in Air at Atmospheric Pressures," FOA contract 96-2507/S summary, (unpublished), November 1996.

[9] L. G. Christophorou and L. A. Pinnaduwage, "Basic Physics of Gaseous Dielectrics," IEEE Transactions on Electrical Insulation, Vol. 25, No. 1, pp. 5574, February 1990.

[10] G. F.Edmiston, "Investigation of Dielectric Window Flashover Under Pulsed High Power Microwave Excitation," Dissertation for the Degree of Doctor of Philosophy in Electrical Engineering, Texas Tech University, Lubbock, Texas, May 2008.

[11] A. Yanguas-Gil, J. Cotrino, L. L. Alves, "An Update of Argon Inelastic CrossSections for Plasma Discharges," Journal of Applied Physics, Vol. 38, pp. 15881598, 2005.

[12] F. Chen, Introduction to Plasma Physics and Controlled Fusion: Volume 1: Plasma Physics, Second Edition, Springer, New York, 2006.

[13] H. C. Kim, and J. P. Verboncoeur, "Time-dependent physics of a single-surface multipactor discharge," Physics of Plasmas, Vol. 12, 123504, 2005. 
[14] H. C. Kim and J. P. Verboncoeur, "Transition of window breakdown from vacuum multipactor discharge to rf plasma," Physics of Plasmas, Vol. 13, 123506, 2006.

[15] D. J. Hemmert, "Experimental Studies of High Power Microwave Breakdown at a Dielectric Surface," Dissertation for the Degree of Doctor of Philosophy in Electrical Engineering, Texas Tech University, Lubbock, Texas, August 2002.

[16] A. Neuber, J. Dickens, D. Hemmert, H. Krompholz, L. L. Hatfield, M. Kristiansen, "Window Breakdown Caused by High-Power Microwaves," IEEE Transactions on Plasma Science, Vol. 26, No. 3, pp.296-303, June 1998.

[17] A. Neuber, D. Hemmert, H. Krompholz, L. Hatfield, M. Kristiansen, "Initiation of High Power Microwave Dielectric Interface Breakdown," Journal of Applied Physics, Vol. 86, No. 3, pp.1724-1728, August 1999.

[18] A. Neuber, D. Hemmert, J. Dickens, H. Krompholz, L. Hatfield, M. Kristiansen, "High Power Microwave Window Breakdown under Vacuum and Atmospheric Conditions," Proceedings of the SPIE Conference, Aerosense 2000, 24-28, April 2000, Orlando, Florida.

[19] D. Hemmert, A. A. Neuber, J. Dickens, H. Krompholz, L. L. Hatfield, M. Kristiansen, "Microwave Magnetic Field Effects on High-Power Microwave Window Breakdown," IEEE Transactions on Plasma Science, Vol. 28, No. 3, pp.472-477, June 2000.

[20] S. E. Calico, "High-Power Microwave Breakdown of Dielectric Interfaces," Dissertation for the Degree of Doctor of Philosophy in Electrical Engineering, Texas Tech University, Lubbock, Texas, August 1991.

[21] J. T. Krile, A. A. Neuber, J.C. Dickens, H. G. Krompholz, "DC Flashover of a Dielectric Surface in Atmospheric Conditions," IEEE Transactions on Plasma Science, Vol. 32, No. 5, pp.1828-1834, October 2004.

[22] K. P. Morales, J. T. Krile, A. A. Neuber, and H. G. Krompholz, "Pulsed Dielectric Surface Flashover in Atmospheric Conditions," IEEE Transactions on Dielectrics and Electrical Insulation, Vol. 13, No. 4, pp.802-809, August, 2006.

[23] J. T. Krile, A. A. Neuber, and H. G. Krompholz, "Monte Carlo Simulation of High Power Microwave Window Breakdown at Atmospheric Conditions," Applied Physics Letters, Vol. 89, 201501, November 2006.

[24] J. Krile, G. Edmiston, K. Morales, A. Neuber, H. Krompholz, M. Kristiansen, "Similarities of Dielectric Surface Flashover under Atmospheric Conditions for Pulsed Unipolar and RF Excitation," Laser Physics, Vol. 16, No. 1, pp.194-201, 2006. 
[25] G. Edmiston, High Power Microwave Window Flashover at Atmospheric Pressures, Thesis for the Degree of Master of Science in Electrical Engineering, Texas Tech University, Lubbock, Texas, December 2005.

[26] G. Edmiston, J. Krile, A. Neuber, J. Dickens, and H. Krompholz, "High Power Microwave Surface Flashover of a Gas-Dielectric Interface at 90 to 760 Torr, IEEE Transactions on Plasma Science, Vol. 34, No. 5, 1782-1788, October 2006.

[27] A. A. Neuber, G. F. Edmiston, J. T. Krile, H. Krompholz, J. C. Dickens, and M. Kristiansen, "Interface Breakdown During High-Power Microwave Transmission,” IEEE Transactions on Magnetics, Vol. 43, No. 1, pp.496-500, January 2007.

[28] G. Edmiston, A. Neuber, L. McQuage, J. Krile, H. Krompholz, J. Dickens, "Contributing Factors to Window Flashover under Pulsed High Power Microwave Excitation at High Altitude," IEEE Transactions on Dielectrics and Electrical Insulation, Vol. 14, No. 4, pp.783-789, August 2007.

[29] G. F. Edmiston, J. T. Krile, and A. A. Neuber, "Imaging of High Power Microwave Induced Surface Flashover on Corrugated Dielectric Window," IEEE Transactions on Plasma Science, $5^{\text {th }}$ Triennial Plasma Imaging Issue, March 2008.

[30] M. B. Lara, J. Mankowski, J. Dickens, M. Kristiansen, "Reflex-triode Geometry of the Virtual-Cathode Oscillator," $14^{\text {th }}$ IEEE Int. Pulsed Power Conference, pp.1161-1164, 2003.

[31] Y. J. Chen "Compact, Repetitive Marx Generator and HPM Generation with the Vircator," Thesis for the Degree of Master of Science in Electrical Engineering, Texas Tech University, Lubbock, Texas, December 2005.

[32] Y. J. Chen, A. A. Neuber, J. Mankowski, J. C. Dickens, M. Kristiansen, R. Gale, "Design and Optimization of a Compact, Repetitive, High-Power Microwave System," Beams Conference, pp.905-908, 2000.

[33] H. Bluhm, Pulsed Power Systems: Principles and Applications, Springer, Berlin, 2006.

[34] J. H. Yee, D. J. Mayhall, G. E. Sieger, R. A. Alvarez, "Propagation of Intense Microwave Pulses in Air and in a Waveguide," IEEE Transactions on Antennas and Propagation, Vol. 39, No. 9, pp.1421-1427, September 1991.

[35] U. S. Inan, A. S. Inan, Electromagnetic Waves, Prentice Hall, New Jersey, 2000.

[36] D. A. Bathker, "Simple Rectangular to Circular Microwave Waveguide Transitions," http://www.hamradio.com/sbms/techpapers/K6BLG/circ_rect.html, 2004. 
[37] L. McQuage, G. Edmiston, J. Mankowski, A. Neuber, "Short Pulse High Power Microwave Surface Flashover," IEEE $34^{\text {th }}$ International Conference on Plasma Science, pp. 258-258, 2007.

[38] H. G. Krompholz, L. L. Hatfield, A. A. Neuber, K. P. Kohl, J. E. Chaparro, H. Y Ryu, "Phenomenology of Subnanosecond Gas Discharges at Pressures Below One Atmosphere," IEEE Transactions on Plasma Science, Vol. 34, pp. 927-937, 2007.

[39] J. E. Chaparro, W. J. Justic, H. G. Krompholz, L. L. Hatfield, A. A. Neuber, "Breakdown Delay Times for Subnanosecond Gas Discharges at Pressures Below One Atmosphere,” IEEE Transactions on Plasma Science, 2008.

[40] J. E. Chaparro, "Investigation of Sub-Nanosecond Breakdown through Experimental and Computational Methods," Dissertation for the Degree of Doctor of Philosophy in Electrical Engineering, Texas Tech University, Lubbock, Texas, August 2008. 


\section{Appendix A \\ Circular Waveguide Design and Calculations}

The circular WG described in Sec. 3.2.2 was designed with the goal of ensuring single mode propagation over a chosen range of frequencies. This range is bounded by the cutoff frequency for the dominant $\mathrm{TE}_{11}$ mode, below which no propagation occurs, and that for the higher order $\mathrm{TM}_{01}$ mode, above which the higher order mode may propagate as well. The center frequency for this range was chosen to be $3 \mathrm{GHz}$ based on previous work by Chen [31]. The cutoff frequencies for the $\mathrm{TE}_{11}$ and $\mathrm{TM}_{01}$ modes are given by [35]:

$$
\begin{aligned}
& f_{c T E 11}=\frac{s_{11} \cdot c}{2 \cdot \pi \cdot r} \\
& f_{c T M 01}=\frac{t_{01} \cdot c}{2 \cdot \pi \cdot r}
\end{aligned}
$$

where $r$ is the radius of the WG, $c$ is the speed of light, and $s_{11}$ and $t_{01}$ are Bessel function roots equal to 1.841 and 2.405 respectively. Cutoff frequencies for several commercially available pipe sizes were calculated to determine which would be the best fit and are shown in Table 6.1.

\section{Table 6.1: WG Cutoff Frequencies}

$\begin{array}{cccccc}\begin{array}{c}\text { Nominal Size } \\ \text { [in] }\end{array} & \text { Schedule } & \begin{array}{c}\text { Inner Diameter } \\ {[\mathrm{in}]}\end{array} & \begin{array}{c}\text { Radius } \\ {[\mathrm{cm}]}\end{array} & \mathrm{f}_{\text {CTE11 }}[\mathrm{GHz}] & \mathrm{f}_{\text {CTM01 }}[\mathrm{GHz}] \\ 3 & 10 & 3.26 & 4.14 & 2.123 & 2.774 \\ 3 & 40 & 3.068 & 3.896 & 2.256 & 2.947 \\ 3 & 80 & 2.9 & 3.683 & 2.387 & 3.118 \\ 2.5 & 10 & 2.635 & 3.347 & 2.625 & 3.431 \\ 2.5 & 40 & 2.469 & 3.136 & 2.803 & 3.662 \\ 2.5 & 80 & 2.323 & 2.95 & 2.98 & 3.892\end{array}$

Although the desired $3 \mathrm{GHz}$ falls within several of the above ranges, the 2.5", Sch. 10 pipe was chosen because its center frequency lies closest to $3 \mathrm{GHz}$. With the radius now chosen to be $3.347 \mathrm{~cm}$, the propagation constant, group velocity, and WG impedance for the dominant mode can be calculated by [Inan]: 


$$
\begin{aligned}
& \bar{\beta}_{T E 11}=\sqrt{\omega^{2} \mu \varepsilon-\left(\frac{s_{11}}{r}\right)^{2}} \\
& \bar{v}_{g 11}=c \sqrt{1-\left(\frac{f_{c T E 11}}{f}\right)^{2}} \\
& Z_{T E 11}=\frac{\eta}{\sqrt{1-\left(\frac{f_{c T E 11}}{f}\right)^{2}}}
\end{aligned}
$$

which were found to be $30.441 / \mathrm{m}, 1.451 * 10^{8} \mathrm{~m} / \mathrm{s}$, and $778 \Omega$ respectively. 


\section{APPENDIX B}

\section{Rectangular to Circular WG Transition Design}

In order to excite the WG with a known signal during calibration, a coaxial to WG transition is needed, but since they are not commercially available for circular WGs, a rectangular to circular WG transition is also needed. An Omni Wave MST-69 coaxial to WR-284 WG transition was chosen because its frequency range of operation most closely overlaps that of the circular WG. The design method for the rectangular to circular WG transition was based on [36], but with some differences. In that method, a standard WG is used to determine the dimensions for a circular WG and a quarter-wave transition such that the WG wavelengths are the same for all three sections. The impedance of the transition is also designed to be the geometric mean of the rectangular and circular impedances and it is a quarter-wavelength long so that the waves reflected from each end of the transition are $180^{\circ}$ out of phase and destructively interfere with each other. Since the dimensions for the circular WG are already set, this method is somewhat altered, but with the same goal of achieving WG wavelengths as close as possible. The cutoff wavelengths for the circular and rectangular WGs are given by []:

$$
\begin{aligned}
& \lambda_{\text {c.circ }}=\frac{2 \cdot \pi \cdot r_{\text {circ }}}{s_{11}} \\
& \lambda_{\text {c.rect }}=2 \cdot a_{\text {rect }}
\end{aligned}
$$

where $\mathrm{r}_{\text {circ }}$ is the radius of the circular WG and $\mathrm{a}_{\mathrm{rect}}$ is the width of the rectangular WG. If the radius of the circular WG had not been previously determined, then it could be found by setting these equations equal to each other and solving for the circular WG radius, in which case the WG wavelengths would be equal for any frequency. Since these dimensions are already set, they can be readily calculated and are shown in Table 6.2. 
Table 6.2: WG Transition Values

$\begin{array}{ccccccc} & \mathrm{a}[\mathrm{in}] & \mathrm{b}[\mathrm{in}] & \mathrm{r}[\mathrm{in}] & \lambda . \mathrm{c}[\mathrm{in}] & \begin{array}{c}\text { Relative } \\ \text { Impedance }\end{array} & \lambda . \mathrm{WG} \text { [in] } \\ \text { Circular WG } & \mathrm{N} / \mathrm{A} & \mathrm{N} / \mathrm{A} & 1.318 & 4.496 & 2 & 8.128 \\ \text { Rectangular WG } & 2.84 & 1.34 & \mathrm{~N} / \mathrm{A} & 5.68 & 0.944 & 5.455 \\ \text { Transition } & 2.736 & 1.879 & \mathrm{~N} / \mathrm{A} & 5.471 & 1.374 & 5.662\end{array}$

The dimensions of the transition are determined by setting its impedance such that the Eq. 6.8 is satisfied.

$$
Z_{\text {tran }}=\sqrt{Z_{\text {circ }} \cdot Z_{\text {rect }}}
$$

Instead of using more complicated expressions for WG impedance, a simplified relative impedance is used, given by:

$$
Z=2 \cdot \frac{b}{a}
$$

For a circular $\mathrm{WG}$, this expression simplifies to 2 , since $\mathrm{a}$ and $\mathrm{b}$ are both equal to $2 * \mathrm{r}$. For the WR-284, the relative impedance is equal to 0.944 , since the width of the WG is nearly twice the height. Since there are still two unknown dimensions, the width of the transition is determined by setting it to the geometric mean of the circular and rectangular widths.

$$
b_{\text {tran }}=\sqrt{b_{\text {rect }} \cdot 2 r_{\text {circ }}}
$$

Now the height of the transition can be determined by setting Eq. 6.8 and Eq. 6.9 equal to each other and rearranging:

$$
a_{\text {tran }}=\frac{2 b_{\text {tran }}}{\sqrt{Z_{\text {circ }} \cdot Z_{\text {rect }}}}
$$

The length of the transition is a quarter of the WG wavelength, but since it is not the same for each section, it was calculated for each section by:

$$
\bar{\lambda}_{W G}=\frac{\lambda_{0}}{\sqrt{1-\left(\frac{\lambda_{0}}{\lambda_{c}}\right)^{2}}}
$$

where $\lambda_{0}$ is the wavelength in free space at $3 \mathrm{GHz}$. Transition designs were then simulated in HFSS to determine the optimal length, which was found to be $1.56 \mathrm{in}$. A plot of simulated scattering parameters over a frequency range is shown in Fig. 6.1. 


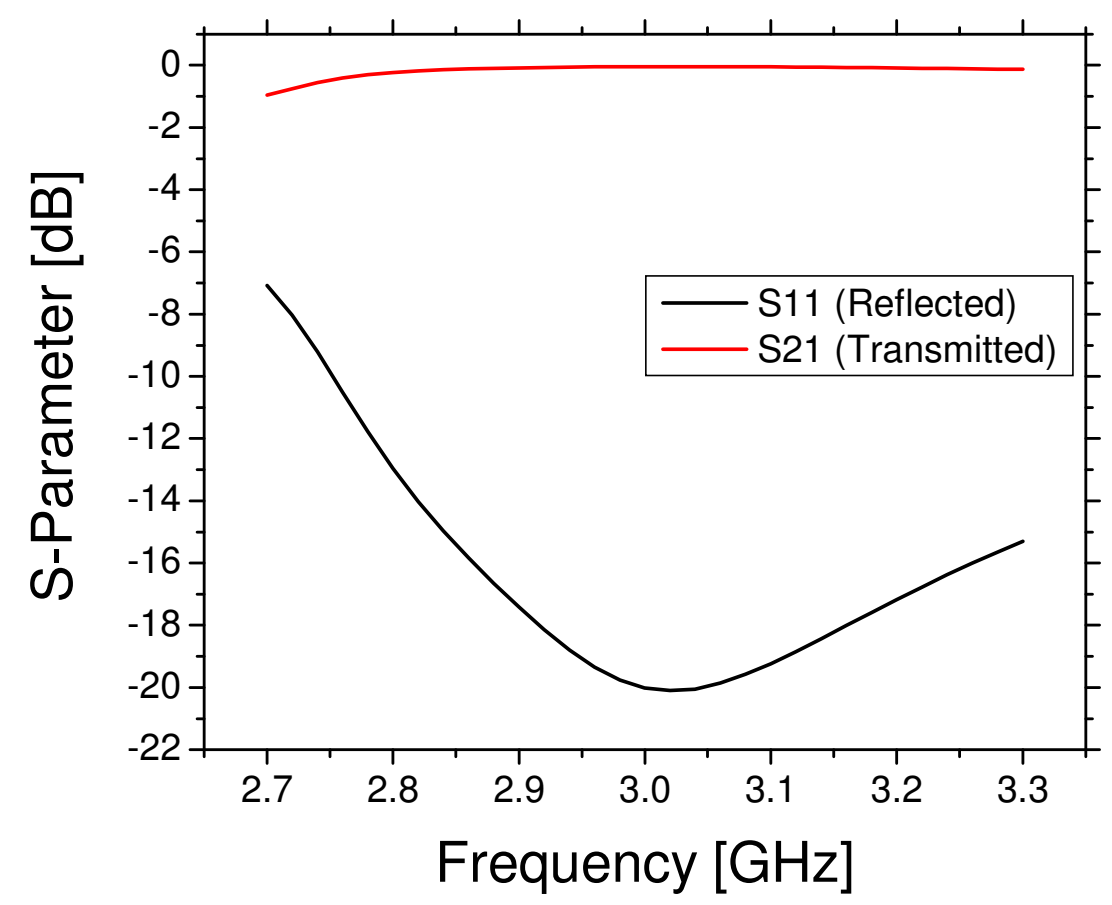

Figure 6.1: R/C Transition S-Parameters 


\section{PERMISSION TO COPY}

In presenting this thesis in partial fulfillment of the requirements for a master's degree at Texas Tech University or Texas Tech University Health Sciences Center, I agree that the Library and my major department shall make it freely available for research purposes. Permission to copy this thesis for scholarly purposes may be granted by the Director of the Library or my major professor. It is understood that any copying or publication of this thesis for financial gain shall not be allowed without my further written permission and that any user may be liable for copyright infringement.

Agree (Permission is granted.)

Student Signature

Disagree (Permission is not granted.)

Luke McQuage

Student Signature
Date

$12 / 3 / 2008$

Date 